\title{
Spectrum Pricing Games with Bandwidth Uncertainty and Spatial Reuse in Cognitive Radio Networks
}

\author{
Gaurav S. Kasbekar \\ Department of Electrical and Systems \\ Engineering \\ University of Pennsylvania \\ kgaurav@seas.upenn.edu
}

\author{
Saswati Sarkar \\ Department of Electrical and Systems \\ Engineering \\ University of Pennsylvania \\ swati@seas.upenn.edu
}

\begin{abstract}
In cognitive radio networks (CRN), primary users can lease out their unused bandwidth to secondary users in return for a fee. We study price competition in a CRN with multiple primaries and multiple secondaries in a region, where each primary tries to attract secondaries by setting a lower price for his bandwidth than other primaries. A CRN has two distinctive features, which makes the price competition very different from that in traditional commodity markets. First, in every slot, each primary may or may not have unused bandwidth available. So primaries are uncertain about the number of other primaries from whom they face competition. Second, spectrum is a commodity that allows spatial reuse: the same band can be simultaneously used at far-off locations without interference; on the other hand, simultaneous transmissions at neighboring locations on the same band interfere with each other. As a result, a primary cannot offer bandwidth at all locations, but must select an independent set of locations at which to offer it. Also, the choice of the independent set and the prices at those locations must be made jointly. We formulate price competition in a CRN as a game, taking into account both bandwidth uncertainty and spatial reuse. We analyze the game in a single slot, as well as its repeated version. In each case, we not only prove the existence of a Nash equilibrium, but also explicitly compute it. The expressions we obtain provide interesting insights into how the price competition evolves for different values of the system parameters. Moreover, for the game in a single slot, we prove the uniqueness of the Nash equilibrium in the class of symmetric equilibria.
\end{abstract}

\section{Introduction}

The last decade has seen a tremendous growth in wireless networks, resulting in a proportionate increase in demand for spectrum. But spectrum is limited, which has led to the design of techniques such as Cognitive Radio Technology [2], for using the available spectrum more efficiently. In Cognitive Radio Networks (CRNs), there are two types of spectrum users: (i) a primary user who leases a certain portion (channel or band) of the spectrum directly from the regulator, and (ii) secondary users who can use the channel when it is not used by the primary.

We consider a CRN with multiple primary and sec- ondary users in a region. Time is slotted, and in every slot, each primary has unused bandwidth with some probability, which he would like to sell to secondaries. Now, secondaries would like to buy bandwidth from the primaries that offer it at a low price, which results in price competition among the primaries. If a primary quotes a low price, it will attract buyers, but at the cost of reduced revenues. This is a common feature of an oligopoly [1], in which multiple firms sell a common good to a pool of buyers. Price competition in an oligopoly is naturally modeled using game theory [15], and has been extensively studied in economics using for example the classical Bertrand game [1] and its variants.

However, a CRN has several distinguishing features, which makes the price competition very different from oligopolies encountered in economics. First, in every slot, each primary may or may not have unused bandwidth available. So a primary who has unused bandwidth is uncertain about the number of primaries from whom he will face competition. A low price will result in unnecessarily low revenues in the event that very few other primaries have unused bandwidth, because even with a higher price the primary's bandwidth would have been bought, and vice versa. Second, spectrum is a commodity that allows spatial reuse: the same band can be simultaneously used at far-off locations without interference; on the other hand, simultaneous transmissions at neighboring locations on the same band interfere with each other. Thus, spatial reuse provides an opportunity to primaries to increase their profit by selling the same band to secondaries at different locations, which they can utilize subject to satisfying the interference constraints. So when multiple primaries own bandwidth in a large region, each needs to decide on a set of non-interfering locations (called independent set (I.S.)) within the region, at which to offer bandwidth. This is another source of strategic interaction among the primaries- each primary would like to select a maximum-sized I.S. to offer bandwith at; but if a lot of primaries offer bandwidth at the same locations, there is intense competition at those locations. So a primary would have benefited by instead offering bandwidth at a smaller I.S. and charging high prices at those locations. To the best of our knowledge our work is the first to consider either of these distinguishing features in context of price competition in wireless networks. 
We model the problem using game theory, and our model captures both uncertain bandwidth availability and spatial reuse. We first consider (i) a one-shot game, in which bandwidth trading is done only once, and subsequently (ii) a repeated game in which there are an infinite number of slots, and bandwidth trading is done every slot. We analyze the games at a single location, and at multiple locations with spatial reuse constraints, and seek a Nash equilibrium [15] (NE) in each case.

In the one-shot game at a single location (Section 3), we show that there does not exist a pure-strategy NE, i.e., one in which each primary deterministically selects a price (Section 3.3). This is in sharp contrast with the Bertrand game [1], where each seller always has his ware available - the only equilibrium then is a pure-strategy one in which each seller chooses the lowest possible price [8]. We then explicitly find a mixed-strategy NE in which each primary randomly chooses a price from a range, and prove that it is unique in the class of symmetric equilibria (Section 3.3). As the probability that a primary has bandwidth available decreases, this range of prices becomes increasingly concentrated at the highest possible price. This confirms the intuition that when spectrum holes are rarely available, whenever a primary has a spectrum hole, he can afford to set a high price in view of the limited competition he anticipates from others. Using the explicit expressions, we quantify the loss of total revenue incurred due to competition under symmetric equilibria (Section 3.4). Our numerical computations reveal that this loss, or equivalently, the efficiency of the symmetric equilibria, exhibits interesting threshold behavior, which we also analytically prove in the asymptotic regime (i.e., when the number of primaries is large).

Next, we consider a one-shot game when the primaries offer bandwidth at multiple locations arranged in a line and also in a square grid (Fig. 3) (Section 4), and prove that there exists a unique symmetric NE which we explicitly compute (Sections 4.1,4.2). When the number of such locations is even, there are two disjoint I.S. of maximum size, and we prove that at the equilibrium each primary selects between the two maximum I.S. with equal probability (Sections 4.1.1,4.2). When the number of locations is odd, there exists a unique maximum I.S., and a slightly smaller one. We show that when the bandwidth availability probability $q$ is smaller than a threshold, all primaries offer bandwidth only at the maximum I.S. at equilibrium (Sections 4.1.2.1,4.2). This is because even though a primary would be able to charge a high price at each node by unilaterally deviating to the smaller I.S., he prefers to stay at the maximum I.S. because the latter is larger and the competition is likely to be limited. But when $q$ exceeds the threshold, primaries randomize between offering bandwidth at the maximum and second largest I.S. (Sections 4.1.2,4.2). We explicitly compute the above threshold, the probabilities of the above selection as also the distribution for selecting the price at each node in the selected independent set for both odd and even number of locations (Sections 4.1.1,4.1.2,4.2).

Next, we analyze the repeated game version of the one-shot game at a single location (Section 5), and show that there exists an efficient NE in which each primary sets the highest possible price and as a result, the sum of expected revenues of the primaries is maximized. This is achieved through a threat mechanism: if any primary lowers his price in a slot, all others retaliate in future slots by playing the one-shot game NE strategy and hence the primary suffers in the long run.

Our main contribution is that we are able to explicitly compute NE in all the games we consider. Since the prices can take real values, the strategy sets of players are continuous. Thus, classical results do not establish existence and uniqueness of $\mathrm{NE}$ for the games we consider, and there is no standard algorithm for finding a NE, unlike when each player's strategy set is finite [15]. The explicit computations provide valuable insight; in particular, they clearly reveal the effect of the system parameters on equilibrium behavior. All proofs are deferred to the Appendix.

\section{Related Work}

Pricing related issues have been extensively studied in the context of wired networks and the Internet; see [7] for an overview. Price competition among spectrum providers in wireless networks has been studied in [16], [17], [18], [19], [20], [21]. Specifically, Niyato et. al. analyze price competition among multiple primaries in CRNs [20], [21]. However, neither uncertain bandwidth availability, nor spatial reuse is modeled in any of the above papers. Also, most of these papers do not explicitly find a NE (exceptions are [17], [20]). Our model incorporates both uncertain bandwidth availability and spatial reuse, which makes the problem challenging; despite this, we are able to explicitly compute a NE. Zhou et. al. [22] have designed double auction based spectrum trades in which an auctioneer chooses an allocation taking into account spatial reuse and bids. However, in the price competition model we consider, each primary independently sells bandwidth, and hence a central entity such as an auctioneer is not required.

In the economics literature, the Cournot game and the Bertand game are two basic models that have been widely used to study competition among sellers in oligopolies [1]. In a Cournot game, sellers choose the quantity of a good to produce as opposed to prices in a Bertrand game, and hence the latter is more relevant to our model. In a Bertrand game, each seller quotes a price for a good, and the buyers buy from the seller that quotes the lowest price ${ }^{1}$ [1]. Several variants of the Bertrand game have been studied, e.g., [4], [5], [6], [25]. Osborne et al [4] consider price competition in a duopoly, when the capacity of each firm is constrained. Chawla et. al. [25] consider price competition in networks where each seller owns a capacity-constrained link, and decides the price for using it; the consumers choose paths they would use in the networks based on the prices declared and pay the sellers accordingly. The capacities in both

\footnotetext{
${ }^{1}$ If two or more sellers quote the lowest price, the demand is equally shared between them.
} 
cases are deterministic, whereas the availability of bandwidth is random in our model. The work most closely related to ours is the paper by Janssen et al [6], which analyze the case where each seller may be inactive with some probability. However, none of the above papers [4], [5], [6], [25] consider the spectrum-specific issue of spatial reuse, which introduces a new dimension, that each player not only needs to determine the price of the commodity he owns (as in [4], [5], [6], [25]), but also select an independent set to compete in. The joint decision problem significantly complicates the analysis. Also, the results in [6] are restricted to the case of one buyer; but, a CRN is likely to have multiple secondaries, which our model allows. This again complicates the analysis since multiple primaries can now sell their available bandwidths. Finally, unlike [6], we consider repeated interactions among primaries, unequal probabilities of availability of unused bandwidth, random valuations for secondaries (Sections 5,6.1, 6.2).

\section{Price Competition at a Single Location 3.1 Model}

Suppose there are $n \geq 2$ primaries and $k \geq 1$ secondaries in a region. Each secondary may constitute a customer who requires 1 unit of bandwidth, or may simply be a demand for 1 unit of bandwidth. We initially consider the case that the primaries know $k$, and later generalize our results to allow for random, and apriori unknown, $k$. Time is divided into slots of equal duration. In every slot, each primary has 1 unit of unused bandwidth with probability $q$ and 0 units with probability $1-q$, where $0<q<1$. We initially assume that the bandwidth availability probability $q$ is the same for all primaries, but subsequently allow unequal probabilities in Section 6.1. A primary $i$ who has unused bandwidth in a slot can lease it out to a secondary for the duration of the slot, in return for an access fee of $p_{i}$. Leasing in a slot incurs a cost of $c \geq 0$. This cost may arise, for example, if the secondary uses the primary's infrastructure to access the Internet. We assume that $p_{i} \leq v$ for each primary, for some constant $v>c$. This upper bound $v$ may arise as follows:

1. The spectrum regulator may impose this upper bound to ensure that primaries do not excessively overprice bandwidth even when competition is limited owing to bandwidth scarcity or high demands from secondaries, or when the primaries collude.

2. Alternatively, the valuation of each secondary for 1 unit of bandwidth may be $v$, and no secondary will buy bandwidth at a price that exceeds his valuation. We initially assume that the primaries know this upper limit $v$, which is likely to be the case for the first interpretation. For the second interpretation, the primaries need not know the secondaries' valuations, - we consider this generalization in Section 6.2.

Secondaries buy bandwidth from the primaries that offer the lowest price. More precisely, in a given slot, let $Z$ be the number of primaries who offer unused bandwidth. Then the bandwidth of the $\min (Z, k)$ primaries that offer the lowest prices is bought (ties are resolved at random).

\subsection{Game Formulation}

We formulate the above price competition among primaries as a game, which is any situation in which multiple individuals called players interact with each other, such that each player's welfare depends on the actions of others [1]. In our model, the primaries are the players, and the action of primary $i$ is the price $p_{i}$ that he chooses $^{2}$. In Sections 3, 4, we study the interaction of the primaries in a single slot, which is referred to as the one-shot game. In Section 5, we consider a setting where the one-shot game is repeated an infinite number of times, which is referred to as the repeated game.

The utility or payoff of a player in a game is a numerical measure of his satisfaction level [1], which in our context is the corresponding primary's net revenue. In (the one-shot version of) our game, the utility of primary $i$ is 0 if he has no unused bandwidth. Let $u_{i}\left(p_{1}, \ldots, p_{n}\right)$ denote his utility if he has unused bandwidth ${ }^{3}$ and primary $j$ sets a price of $p_{j}, j=1, \ldots, n$. Thus,

$u_{i}\left(p_{1}, \ldots, p_{n}\right)= \begin{cases}p_{i}-c & \text { if primary } i \text { sells his bandwidth } \\ 0 & \text { otherwise }\end{cases}$

Recall that the distribution function (d.f.) [24] of a random variable (r.v.) $X$ is the function:

$$
G(x)=P(X \leq x), x \in R
$$

where $R$ is the set of real numbers. Now, a strategy [1] for primary $i$ is a plan for choosing his price $p_{i}$. We allow each primary $i$ to choose his price randomly from a set of prices using an arbitrary d.f. $\psi_{i}($.$) , which is referred to as$ the strategy of primary $i$. A d.f. that concentrates its entire mass on a single value allows a primary to deterministically choose this value as his price - such a $\psi($.$) is re-$ ferred to as a pure strategy. The vector $\left(\psi_{1}(),. \ldots, \psi_{n}().\right)$ of strategies of the primaries is called a strategy profile [1]. Let $\psi_{-i}=\left(\psi_{1}(),. \ldots, \psi_{i-1}(),. \psi_{i+1}(),. \ldots, \psi_{n}().\right)$ denote the vector of strategies of primaries other than $i$. Let $E\left\{u_{i}\left(\psi_{i}(),. \psi_{-i}\right)\right\}$ denote the expected utility of player $i$ when he adopts strategy $\psi_{i}($.$) and the other play-$ ers adopt $\psi_{-i}$.

A Nash equilibrium (NE) is a strategy profile such that no player can improve his expected utility by unilaterally deviating from his strategy [1]. Thus, $\left(\psi_{1}^{*}(),. \ldots, \psi_{n}^{*}().\right)$ is a NE if for each primary $i$ :

$$
E\left\{u_{i}\left(\psi_{i}^{*}(.), \psi_{-i}^{*}\right)\right\} \geq E\left\{u_{i}\left(\tilde{\psi}_{i}(.), \psi_{-i}^{*}\right)\right\}, \forall \tilde{\psi}_{i}(.)
$$

When players other than $i$ play $\psi_{-i}, \psi_{i}^{*}($.$) maximizes i$ 's expected utility and is thus his best-response [1] to $\psi_{-i}$.

\subsection{Nash Equilibria}

If $k \geq n$, then the number of buyers is always greater than or equal to the number of sellers. So a primary $i$

\footnotetext{
${ }^{2}$ If primary $i$ has no unused bandwidth, it does not matter what price $p_{i}$ he sets. Yet, for convenience, we speak of $p_{i}$ as being his action.

${ }^{3}$ If instead, $u_{i}\left(p_{1}, \ldots, p_{n}\right)$ were defined to be primary $i$ 's net revenue, unconditional on whether he has unused bandwidth or not, then the expected utilities in the one-shot game analysis would all be scaled by $q$.
} 
will sell his unused bandwidth even when he chooses the maximum possible price $v$. So the strategy profile under which all primaries deterministically choose the price $v$ is the unique NE. So henceforth, we assume that $k \leq n-1$. THEOREM 1. There is no pure strategy NE (i.e., one where every primary selects his price deterministically) in the above game.

In contrast, in the Bertrand game, which corresponds to $q=1$ in our model, the pure strategy profile under which each primary deterministically selects $c$ as his prices is the unique NE [1]. This strategy profile is not a $\mathrm{NE}$ in our context as this provides 0 utility for each primary, whereas by quoting any price above $c$ (and below $v$ ) each primary can attain a positive utility since he will sell his unused bandwidth at least when he is the only primary that has unused bandwidth which happens with positive probability (since $q<1$ ). We have shown that no other deterministic strategy profile is a NE either.

Next, we focus on a specific class of Nash equilibria, known as symmetric Nash equilibria. A NE $\left(\psi_{1}^{*}(),. \ldots, \psi_{n}^{*}().\right)$ is a symmetric $\mathrm{NE}$ if all players play identical strategies under it, i.e., $\psi_{1}^{*}()=.\psi_{2}^{*}()=.\ldots=$ $\psi_{n}^{*}($.$) . In practice it is challenging to implement any$ other NE - the simple example of two primaries and a NE of $\left(\psi_{1}^{*}(),. \psi_{2}^{*}().\right)$ elucidates the inherent complications in the current context. If $\psi_{1}^{*}(.) \neq \psi_{2}^{*}($.$) , then since players$ have the same action sets, utility functions and probability of having unused bandwidth (such games are referred to as symmetric games), $\left(\psi_{2}^{*}(),. \psi_{1}^{*}().\right)$ also constitutes a NE. If player 1 knows that player 2 is playing $\psi_{2}^{*}($. $\left(\psi_{1}^{*}(\right.$.$) respectively), he would choose the best response$ $\psi_{1}^{*}().\left(\psi_{2}^{*}(\right.$.$) respectively), but he can not know player 2's$ choice between the two options without explicitly coordinating with him, which is again ruled out due to the competition between the two. Under symmetric NE, all players play the same strategy, and thus this quandary is somewhat limited - symmetric NE has indeed been advocated for symmetric games by several game theorists [3]. The natural question now is whether there exists at least one symmetric NE, and also whether there is a unique symmetric NE (only uniqueness will eliminate the above quandary). Note that some symmetric games are known to have multiple symmetric NE. For example, consider the simple "Meeting in New York game" [1] with two players, where each player can either be at Grand Central or at Empire State Building, and both receive unit utility if they meet and zero utility otherwise. The strategies where each player is at Grand Central, and where each player is at Empire State Building, both constitute symmetric NE. We prove existence of a symmetric NE, by explicitly computing one, and subsequently prove that it is the unique symmetric NE in our context.

We first provide the intuition behind our design of a symmetric NE. To simplify our exposition, we introduce the notion of "pseudo-price" for each primary. The pseudo-price of primary $j, p_{j}^{\prime}$, is the price he selects if he has unused bandwidth; $p_{j}^{\prime}=v+1$ otherwise ${ }^{4}$. Consider primary 1 and let $p_{(k)}^{\prime}$ denote the $k$ 'th smallest pseudoprice among the pseudo-prices of the rest of the primaries, i.e., $p_{j}^{\prime}, j \in\{2, \ldots, n\}$ (which 1 will know only after choosing his price or equivalently pseudo-price). Since the primaries choose their prices randomly and since their bandwidth availabilities are random, $p_{(k)}^{\prime}$ is a random variable, and let $F($.$) be its d.f. If primary 1$ offers a price of $x$, he sells his bandwidth only if $p_{(k)}^{\prime}>x$ (since there are $k$ secondaries who opt for the lowest available prices), which happens with probability $(1-F(x))$; the sale fetches a utility of $x-c$. Then, primary 1's expected utility is $(x-c)(1-F(x))$. Now, under NE, primary 1's price distribution being his best response to those of others, he must attain the same expected utility for the entire range of prices he is randomly choosing his price from, more technically, in the entire support set ${ }^{5}$ of his price distribution; this is because his best response price distribution will never select from the less profitable ones which will not therefore be in its support set. Thus, $(x-c)(1-F(x))$ is the same (i.e., a constant) for all $x$ in the support set for his NE price distribution. Hence, $F(x)$ is fully specified once this constant is known, which we determine by considering $F(v)$. Note that $F(v)$ is the probability that $p_{(k)}^{\prime} \leq v$, which happens when $k$ or more primaries have unused bandwidth (among those in $\{2, \ldots, n\})$; this probability therefore is $w(q, n)$, where:

$$
w(q, n)=\sum_{i=k}^{n-1}\left(\begin{array}{c}
n-1 \\
i
\end{array}\right) q^{i}(1-q)^{n-1-i} .
$$

Thus, $F(v)=w(q, n)$. Hence, the constant in question is $(v-c)(1-F(v))=(v-c)(1-w(q, n))$. Thus, in the support set of $F(),. F(x)=1-\frac{(v-c)(1-w(q, n))}{x-c}$. The $x$ at which $F(x)=0$ provides the lower limit of this support set, which, from the above expression, is:

$$
\tilde{p}=v-w(q, n)(v-c) \text {. }
$$

Thus,

$$
F(x)= \begin{cases}0, & x \leq \tilde{p} \\ \frac{x-\tilde{p}}{x-c}, & \tilde{p}<x \leq v .\end{cases}
$$

We now only need to determine a price d.f. $\psi($.$) for$ each primary that leads to the above d.f. $F($.$) for the k$ th smallest pseudo-price of $n-1$ primaries. Note that the pseudo-price for any given primary is less than or equal to $x$ (where $x \leq v$ ) whenever he has unused bandwidth and he quotes a price of $x$ or less: the probability that both these events occur is $q \psi(x)$. Thus, since $F(x)$ is the probability that $k$ or more pseudo-prices (among those $n-1$ ) are less than or equal to $x, F(x)$ equals

$$
\sum_{i=k}^{n-1}\left(\begin{array}{c}
n-1 \\
i
\end{array}\right)[q \psi(x)]^{i}[1-q \psi(x)]^{n-1-i},
$$

\footnotetext{
${ }^{4}$ The choice $v+1$ is arbitrary. Any other value greater than $v$ would also work.

${ }^{5}$ The support set of a d.f. is the smallest closed set such that its complement has probability zero under the d.f. [24].
} 
for all $x \leq v$. Thus, since we know $F($.$) from (4), we can$ compute $\psi(x)=(1 / q) \phi(x)$, where $\phi(x)$ is the solution of the following equation:

$$
\sum_{i=k}^{n-1}\left(\begin{array}{c}
n-1 \\
i
\end{array}\right)[\phi(x)]^{i}[1-\phi(x)]^{n-1-i}=F(x) .
$$

We can in fact formally prove that:

LEMMA 1. Equation (5) has a unique solution $\phi(x) \in$ $[0,1]$. The function $\phi(x)$ is strictly increasing and continuous on $[\tilde{p}, v]$. Also, $\phi(\tilde{p})=0$ and $\phi(v)=q$.

And, the symmetric NE price d.f. $\psi($.$) is:$

$$
\psi(x)= \begin{cases}0, & x \leq \tilde{p} \\ \frac{1}{q} \phi(x), & \tilde{p}<x \leq v \\ 1, & x \geq v\end{cases}
$$

From the properties of the $\phi($.$) function obtained in$ Lemma $1, \psi(x)$ is a continuous d. ${ }^{6}$.

The above intuitive justification however glosses over some technical, nonetheless important, details: we implicitly assume that $F($.$) is continuous and that the set of$ best responses of a primary is a convex set. In the formal proof, we prove both the above for any symmetric NE and subsequently establish that:

THEOREM 2. The strategy profile in which each primary $i$ chooses his price $p_{i}$ according to $\psi($.$) , where \psi($. is defined by (6), (5), (4) is the unique symmetric NE.

This random selection of prices as per $\psi($.$) can be in-$ terpreted as follows: each primary $i$ sets a base price $v$ and randomly holds "sales" to attract secondaries by lowering the price to some value $p_{i} \in[\tilde{p}, v]^{7}$.

Example: For $n=2$ and $k=1$, we have $w(q, n)=q$, $\tilde{p}=v-q(v-c)$, and

$$
\psi(x)= \begin{cases}0 & x \leq \tilde{p} \\ \frac{1}{q}\left(\frac{x-\tilde{p}}{x-c}\right) & \tilde{p}<x \leq v \\ 1 & x \geq v\end{cases}
$$

REMARK 1. Our results readily generalize to allow for a random number of secondaries $(K)$. Then the primaries apriori know only the probability mass function (p.m.f.) for $K, \operatorname{Pr}(K=k)=\gamma_{k}$, but not the value of $K$. Unlike in (2), we now define $w(q, n)$ as:

$$
w(q, n)=\sum_{k=1}^{n-1} \gamma_{k} \sum_{i=k}^{n-1}\left(\begin{array}{c}
n-1 \\
i
\end{array}\right) q^{i}(1-q)^{n-1-i}
$$

Also, (5) is replaced by:

$$
\sum_{k=1}^{n-1} \gamma_{k} \sum_{i=k}^{n-1}\left(\begin{array}{c}
n-1 \\
i
\end{array}\right)[\phi(x)]^{i}[1-\phi(x)]^{n-1-i}=F(x)
$$

Now, $\psi($.$) computed as before, but with the above modi-$ fications in $w(.,),. \phi($.$) , again constitutes the unique sym-$ metric NE strategy of each primary.

\footnotetext{
${ }^{6} \mathrm{~A}$ function $f(x)$ is a d.f. iff it is increasing, right continuous, and has limits 0 and 1 as $x$ tends to $-\infty$ and $\infty$ respectively [24].

${ }^{7}$ This interpretation has been suggested in [10] for random selection of prices in a different context.
}

\subsection{Performance Evaluation under the Unique Symmetric NE}

We first define the efficiency, $\eta$, of a NE as $\eta=\frac{R_{\mathrm{NE}}}{R_{\mathrm{OPT}}}$, where $R_{\mathrm{NE}}$ is the expected sum of utilities of the $n$ primaries at the NE and $R_{\mathrm{OPT}}$ is the maximum possible (optimal) expected sum of utilities. Note that $R_{\mathrm{OPT}}$ is attained only when all primaries cooperate and each selects the maximum possible price $v$ so as to ensure that bandwidth is always sold at this price. Clearly, $\eta \leq 1$ quantifies the loss in total net revenue incurred owing to lack of cooperation among primaries. Also, owing to its uniqueness, the efficiency of the symmetric NE we obtain quantifies fundamental limits on the performance of symmetric NE.

Now, $R_{\mathrm{OPT}}=E[\min (Z, k)](v-c)$, where $Z$ is the number of primaries who have unused bandwidth $(Z$ is a $\operatorname{Binomial}(n, q)$ r.v.). Also, as discussed in Section 3.3, at the unique symmetric NE, whenever a primary has unused bandwidth, he attains an expected utility of $(v-$ $c)(1-F(v))=(v-c)(1-w(q, n))$ irrespective of the price he offers. Thus, since there are $n$ primaries and each has unused bandwidth w.p. $q, R_{\mathrm{NE}}=n q(1-w(q, n))(v-$ c). Hence,

$$
\eta=\frac{n q(1-w(q, n))}{E[\min (Z, k)]} .
$$

Fig. 1 plots $\eta$ of the symmetric NE that we obtain versus $k$ for three values of $q$. It is interesting to note that $\eta$ exhibits a sharp threshold behavior: for $k$ below (respectively, above) a threshold the efficiency is close to 0 (respectively, 1). Also, this threshold is around $n q$, the expected number of primaries who have free bandwidth. Intuitively, this is because, when the supply $n q$ exceeds the demand $k$ for bandwidth (i.e., $k<n q$ ), there is intense price competition, driving down the equilibrium prices. On the other hand as $k$ increases, $w(q, n)$ decreases, $\tilde{p}$ increases (see (3)), and becomes closer to $v$. Hence, the d.f. $\psi($.$) becomes increasingly concentrated at the high-$ est possible price $v$. Intuitively, this is because, when the demand exceeds the supply, a primary expects to sell even at a high price, and sets his price accordingly. The plots for the density of the unique symmetric NE price distribution for different sets of values of parameters $n, k, q$ reveal the same phenomenon as well (Fig. 2).

In fact, we can analytically establish this threshold behavior for large $n$ :

LEMMA 2. Let $q \in(0,1)$ be fixed.

1. If $k \leq(n-1)(q-\varepsilon)$ for some $\varepsilon>0$, then $\eta \rightarrow 0$ as $n \rightarrow \infty$.

2. If $k \geq(n-1)(q+\varepsilon)$ for some $\varepsilon>0$, then $\eta \rightarrow 1$ as $n \rightarrow \infty$.

\section{Price Competition at Multiple Locations with Spatial Reuse of Spectrum}

We now consider the price competition game when primaries can simultaneously offer bandwidths at multiple locations. Each of the $n$ primaries now owns a channel throughout a large region. The primary's own usage of the channel is such that in every slot, he either uses his channel throughout the region (with probability (w.p.) 


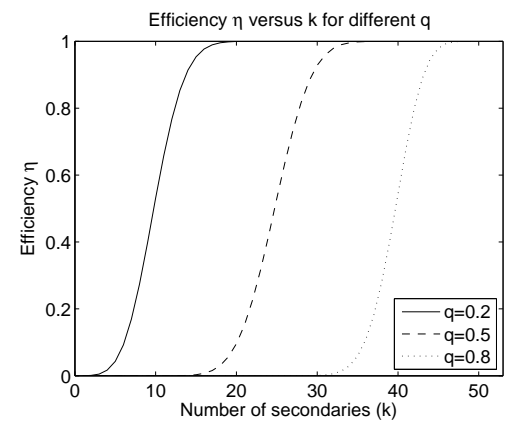

Figure 1. Efficiency of the Nash equilibrium versus $k$ for three values of $q$. The other parameters are $n=50$, $c=0$, and $v=100$.

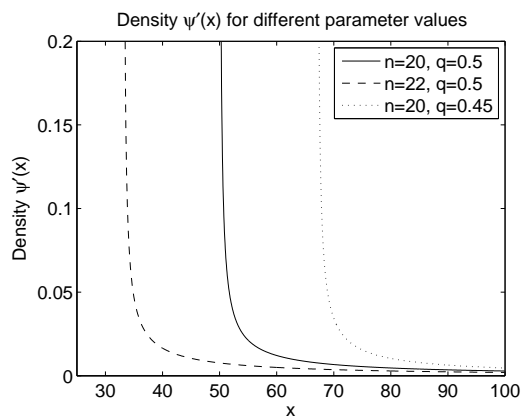

Figure 2. The density $\psi^{\prime}(x)$ for three sets of $n q: 9,10$ and 11 , for the three curves from right to left. The other parameters are $c=0, v=100$ and $k=10$.

$1-q$ ), or does not use it anywhere in the region (w.p. $q$ ). A typical scenario where this happens is when the primary broadcasts the same signal over the entire region, e.g., if the primary is a television broadcaster. Now, the region contains smaller parts, which we refer to as locations. For example, the large region may be a state, and the locations may be towns within it. We initially assume that there are $k$ secondaries at each location, and later outline how the results can be generalized to allow for random and potentially unequal number of secondaries at different locations. As in Section 3, each primary quotes a price of at most $v$, and incurs a cost of $c$ at each location at which it leases bandwidth.

We now describe the spatial reuse constraints that arise owing to simultaneous spectrum usage at multiple locations. The region can be represented by an undirected graph [23] G, called the conflict graph, in which each node represents a location, and there is an edge between two nodes iff transmissions at the corresponding locations interfere with each other. Recall that an independent set [23] (I.S.) in a graph is a set of nodes such that there is no edge between any pair of nodes in the set. Now, a primary who is not using his channel must offer it at a set of mutually non-interfering locations, or equivalently, at an I.S. of nodes; otherwise secondaries ${ }^{8}$ will not be able

\footnotetext{
${ }^{8}$ Note that secondaries are usually customers or local providers, and purchase bandwidth for communication (and not
}

to successfully transmit simultaneously using the bandwidth they purchase, owing to mutual interference. Thus, each primary must jointly select an I.S. at which to offer bandwidth, and the prices to set at the nodes in it. Primaries can randomize their selections over multiple I.S., and also over chosen price ranges.

A strategy of a primary now provides the price distribution he uses at each node and a probability mass function (p.m.f.) for selection among the I. S. (both selections contingent on having unused bandwidth). Note that we allow a primary to use different (and arbitrary) price distributions for different nodes (and therefore allow, but do not require, the selection of different prices at different nodes), and arbitrary p.m.f. (i.e., discrete distributions) for selection among the different I.S. The definitions of $\mathrm{NE}$ and symmetric NE do not change.

We now argue that under any symmetric NE the price distributions at nodes are uniquely specified once the I.S. selection strategy is determined. Let there be $M$ independent sets, and let each primary select among them as per the p.m.f. $\left(r_{1}, \ldots, r_{M}\right)$ (recall that each primary uses the same p.m.f. under a symmetric NE). This provides the probabilities with which a primary offers bandwidth at each node when he has unused bandwidth (this probability for a given node equals the sum of the probabilities associated with all the I.S. that contain the node). Let this selection probability for node $j$ be denoted $\alpha_{j}$. Then, considering that a primary has unused bandwidth w.p. $q$, he offers it at node $j$ w.p. $q \alpha_{j}$. The price selection problem at each node $j$ is now equivalent to that for the single location case investigated in Section 3, the difference being that each primary offers unused bandwidth w.p. $q \alpha_{j}$, instead of $q$, at node $j$. Thus:

LEMma 3. Suppose under a symmetric NE each primary selects node $j$ w.p. $\alpha_{j}$ if he has unused bandwidth. Then under that NE the price distribution of each primary at node $j$ is $\psi($.$) in Section 3.3, with q \alpha_{j}$ in place of $q$. Thus, a symmetric NE strategy is completely specified once the I.S. selection p.m.f. $\left(r_{1}, \ldots, r_{M}\right)$ (which will in turn provide the $\alpha_{j} \mathrm{~s}$ ) is obtained. We determine this p.m.f. for one-dimensional and two-dimensional configurations of nodes.

For a one-dimensional configuration of nodes, we consider a graph $\mathcal{G}$ that is a linear arrangement of nodes as shown in part (a) of Fig. 3 (Section 4.1), and for a two dimensional configuration we allow $\mathcal{G}$ to be a square grid of nodes as shown in part (b) of Fig. 3 (Section 4.2). In both cases, we explicitly compute a symmetric NE, which also turns out to be unique in the class of symmetric NE.

\subsection{Linear Graph}

Let $\mathcal{G}_{m}$ denote a graph that is a linear arrangement of $m \geq 2$ nodes as shown in part (a) of Fig. 3, with an edge between each pair of adjacent nodes. As an example, this would be the conflict graph for locations along a highway or a row of roadside shops. Let the nodes be numbered $1, \ldots, m$ from left to right, and $I_{o}=\{1,3, \ldots\}$ and $I_{e}=$

t.v. broadcasts). Thus, two secondaries can not use the same band simultaneously at interfering locations. 


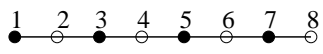

(a) Linear Graph

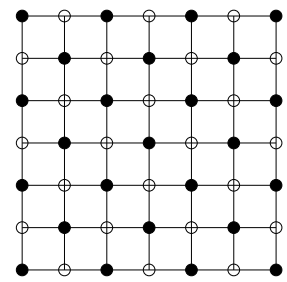

(b) Grid Graph

Figure 3. Part (a) shows a linear graph with $m=8$ and part (b) shows a grid graph with $m=7$. The darkened and un-darkened nodes constitute $I_{o}$ and $I_{e}$ respectively in the linear graph, and $I_{o}^{2}$ and $I_{e}^{2}$ respectively in the grid graph.

$\{2,4, \ldots\}$ be the "odd" and "even" I. S. Note that $I_{o}$ and $I_{e}$ are disjoint I. S., and $I_{o} \cup I_{e}$ is the set of all nodes.

An intuitive property of any NE is that it does not allow a primary to select an I.S. that is not maximal (a maximal I.S. is one that is not a proper subset of any other I.S.). There are however several maximal I.S. in $\mathcal{G}_{m}$, e.g., $\{1,4,6,8, \ldots\}$. The following lemma allows us to rule out all of them except $I_{o}, I_{e}$ under a symmetric NE.

LEMMA 4. A primary never selects any independent set other than $I_{o}$ or $I_{e}$ under a symmetric NE.

The proof relies on showing that if each primary in a given set of $n-1$ primaries selects an I.S. other than $I_{o}, I_{e}$ with positive probability, the remaining primary's best response turns out to be a p.m.f. different from that used by the others - hence a p.m.f. that assigns positive probability to any such I.S. can not constitute a symmetric NE.

Lemma 4 implies that under a symmetric NE the p.m.f. for I.S. selection is characterized by a single probability $t$, with which a primary selects $I_{e}$; each primary selects $I_{o}$ w.p. $1-t$. But, then, each primary selects a node in $I_{e}\left(I_{o}\right.$, resp.) w.p. $t\left(1-t\right.$, resp.). Thus, $\alpha_{j}=t$ if $j \in I_{e}$, and $\alpha_{j}=1-t$ otherwise. We will next compute $t$ under a symmetric NE for the cases $m$ even and odd separately in Sections 4.1.1 and 4.1.2 respectively.

\subsubsection{Even $m$}

Let $m \geq 2$ be even. Then, $\left|I_{o}\right|=\left|I_{e}\right|=\frac{m}{2}$ and both $I_{o}$ and $I_{e}$ have the maximum size among all I.S. (i.e., are maximum I.S.). We can show that $t=1 / 2$ under any symmetric NE. The first step towards that end is to prove that under a symmetric NE each primary selects both $I_{o}$ and $I_{e}$ with positive probability (i.e., $0<t<1$ ). Now, given others' strategies, each primary's expected utility when he selects $I_{o}$ must equal that when he selects $I_{e}$; otherwise $0<t<1$ does not constitute his best response as his utility can be increased by always selecting the one with the larger expected utility. As it turns out, since $\left|I_{o}\right|=\left|I_{e}\right|=\frac{m}{2}$, the two expected utilities are equal if and only if $t=1 / 2$. Thus, under any symmetric NE, a primary competes at any given node w.p. 1/2 (if he has unused bandwidth), i.e., the selection probability for node $j, \alpha_{j}$ is $1 / 2$ for each node $j$. Lemma 3 now provides the price distribution for each primary at each node, and thereby fully specifies a symmetric NE. Formally:

THEOREM 3. The strategy profile in which each primary

- selects $I_{o}$ and $I_{e}$ w.p. 1/2 each, whenever he has unused bandwidth, and

- decides the price at each node in the chosen independent set as per the distribution $\psi($.$) in Sec-$ tion 3.3, with $q$ replaced by $q / 2$ all through, is the unique symmetric $N E$.

\subsubsection{Odd $m$}

Now, let $m \geq 3$ be odd. Then, $\left|I_{o}\right|=\frac{m+1}{2},\left|I_{e}\right|=\frac{m-1}{2}$ and $I_{o}$ is the unique maximum I.S. Each primary now faces the following quandary: (i) whether to offer unused bandwidth only at the nodes in $I_{o}$ and thereby try to sell bandwidth at the maximum possible number of nodes or (ii) to also choose the slightly smaller $I_{e}$ hoping for limited competition there. The answers, and thereby the unique symmetric NE, turn out to be different depending on the value of $w(q, n)$, the probability that $k$ or more of the competitors of any primary have unused bandwidth.

\subsubsection{The Case $w(q, n) \leq \frac{2}{m+1}$ :}

We can prove that under any symmetric $N E$, primaries offer unused bandwidth only in nodes in $I_{o}($ i.e., $t=0$ ). This happens because $w(q, n)$ is so low, that a primary expects to sell his unused bandwidth at each node he offers, and hence he invariably selects the unique maximum I.S. The proof relies on the fact that the best response of any primary, irrespective of the I.S. selection strategies of others is to select $I_{o}$; hence, under any symmetric NE, each primary selects only $I_{o}$. Thus, the selection probability $\alpha_{j}$ for any node $j$ in $I_{o}\left(I_{e}\right.$, resp.) is $1(0$, resp.). Lemma 3 now provides the price distributions at the nodes, and thereby the symmetric NE.

THEOREM 4. Let $m$ be odd and $w(q, n) \leq \frac{2}{m+1}$. The strategy profile in which each primary

- offers unused bandwidth at only the nodes in $I_{o}$, and

- selects the price at each such node according to the d.f. $\psi($.$) in Section 3.3$

is the unique symmetric Nash equilibrium.

REMARK 2. This symmetric NE, which unfortunately is unique, does not allow access to secondary users at nodes in $I_{e}$. But, this scenario is unlikely to arise, especially for large $m$, as this requires very low availability probability $q$.

\subsubsection{The Case $w(q, n)>\frac{2}{m+1}$ :}

Now, $w(q, n)$ is high enough to motivate primaries to offer unused bandwidth in nodes in $I_{e}$ with a positive probability, so as to utilize lower competition there. As before, under any symmetric NE, the value of $t$ must be such that a primary's expected utility when he selects $I_{o}$ must equal that when he selects $I_{e}$. And, the only value of $t$ that attains this equality is the root of the equation (in variable $x$ ),

$$
w(q x, n)(m-1)-w(q(1-x), n)(m+1)+2=0,
$$


which we can prove to be unique in $(0,1)$. Thus, we can prove that under any symmetric $N E, t$ is the unique root in $(0,1)$ of (11). And, since $\alpha_{j}=t$ for $j \in I_{e}, \alpha_{j}=1-t$ for $j \in I_{o}$, we have the price distribution at nodes from Lemma 3. Thus:

THEOREM 5. Let $m$ be odd and $w(q, n)>\frac{2}{m+1}$. The strategy profile in which, whenever a primary has unused bandwidth,

- selects $I_{e}$ and $I_{o}$ w.p. $t$ and $1-t$ respectively, where $t$ is the unique root in $(0,1)$ of $(11)$, and

- selects the price at each node as per distribution $\psi($.$) in Section 3.3, with q replaced by qt (q(1-t)$ resp.) whenever he selects $I_{e}\left(I_{o}\right.$ resp.) constitutes the unique symmetric $N E$.

It can be shown that the selection probability $t$ for $I_{e}$ is less than $1 / 2$, and increases with $m$ and tends to $\frac{1}{2}$ in the limit as $m \rightarrow \infty$. Fig. 4 shows an example. This is because as $m$ grows, the ratio $\frac{\left|I_{e}\right|}{\left|I_{o}\right|}=\frac{m-1}{m+1}$ increases, and converges to 1 . Thus, the symmetric NE converges to that for even $m$. Fig. 4 also shows that for all $m, t$ is higher for $q=0.7$ than for $q=0.5$. This is because for a higher value of $q$, the competition at $I_{o}$ is more intense and hence primaries deviate to $I_{e}$ with a higher probability.

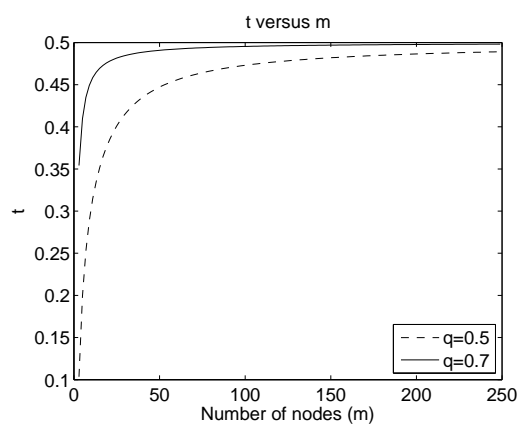

Figure 4. $t$ versus $m$ for $q=0.5$ and $q=0.7$. The other parameter values are $c=0, v=100, n=20, k=9$.

\subsection{Grid Graph}

Now, consider an $m \times m$ grid graph $\mathcal{G}_{m, m}$, in which $m^{2}$ nodes (locations) are arranged in a square grid as shown in part (b) of Fig. 3. There is an edge between each pair of adjacent nodes in the same row or column. For example, this graph may represent a shopping complex, with the nodes corresponding to the locations of shops with Access Points (AP) for Internet access. The transmission ranges of the APs are such that the range of each AP overlaps with those of adjacent APs in the same row and column, and not with those of other APs.

Let $V_{i j}$ denote the node in the $i$ 'th row from the top and the $j$ 'th column from the left. Let:

$$
\begin{aligned}
I_{o}^{2} & =\left\{V_{i j}: i+j \text { is even }\right\} \\
I_{e}^{2} & =\left\{V_{i j}: i+j \text { is odd }\right\} .
\end{aligned}
$$

In part (b) of Fig. 3, the darkened and un-darkened nodes constitute $I_{o}^{2}$ and $I_{e}^{2}$ respectively. Note that $I_{o}^{2}$ and $I_{e}^{2}$ are disjoint I.S. and $I_{o}^{2} \cup I_{e}^{2}$ is the set of all nodes. For even $m$,
$I_{o}^{2}$ and $I_{e}^{2}$ are both maximum I.S. of size $\frac{m^{2}}{2}$. For odd $m$, $I_{o}^{2}$ is the unique maximum I.S. and has size $\frac{m^{2}+1}{2}$; also, $\left|I_{e}^{2}\right|=\frac{m^{2}-1}{2}$.

The NE analysis is similar to that for the linear graph. We simply outline the results.

1. When $m$ is even, the strategy presented in Theorem 3 , with $I_{o}, I_{e}$ replaced by $I_{o}^{2}, I_{e}^{2}$ respectively is the unique symmetric NE.

2. When $m$ is odd, and $w(q, n) \leq \frac{2}{m^{2}+1}$, the strategy presented in Theorem 4, with $I_{o}$ replaced by $I_{o}^{2}$ is the unique symmetric NE.

3 . When $m$ is odd, and $w(q, n)>\frac{2}{m^{2}+1}$, the strategy presented in Theorem 5, with $I_{o}, I_{e}$ replaced by $I_{o}^{2}, I_{e}^{2}$ respectively and (11) used with $m^{2}$ instead of $m$ is the unique symmetric NE.

We end this section with the following remarks which apply for both the linear and the grid arrangements.

REMARK 3. A primary may offer equal prices at all nodes in the I.S. he selects, the price being sampled from the symmetric NE distributions presented so far, or may decide prices independently at each node, again sampled using the above distributions - all our analytical results hold in both cases.

REMARK 4. All our analytical results hold even when the number of secondaries at different nodes are unequal, and not known to the primaries apriori, but constitute statistically identical random variables. The only difference arises in the expressions for $w(q, n)$ and the price distributions $\psi($.$) , which can be modified as indicated in Re-$ mark 1 (starting from those obtained for the corresponding cases with deterministic number of secondaries).

REMARK 5. With the efficiency of a NE defined as in Section 3.4, the efficiency for the NE found in this section exhibit threshold behavior similar to that in Lemma 2.

\section{Price Competition under Repeated Inter- actions}

We now consider repeated interactions among primaries in multiple slots. We first formulate the problem in Section 5.1 and then describe our results in Section 5.2.

\subsection{Formulation}

We consider a repeated game [1] formulation for the one-shot game at a single location ${ }^{9}$ described in Section 3, where the one-shot game is repeated an infinite number of times, at $\tau=1,2,3, \ldots$. Each player perfectly recalls the actions of every player in all preceding times. The payoff of player $i$ for the overall repeated game is defined to be $u_{i}=\sum_{\tau=1}^{\infty} \delta^{\tau-1} u_{i, \tau}$, where $u_{i, \tau}$ is his payoff at time $\tau$ and $\delta \in(0,1)$ is the discount factor [1], which is used to discount future payoffs (see [1], [15] for interpretations of the discount factor). The discount factor is usually close to 1 [1].

A strategy of a player in a repeated game is a complete plan for choosing the action in each slot as a function of

\footnotetext{
${ }^{9} \mathrm{We}$ omit interactions at multiple locations (Section 4) owing to space constraints.
} 
the actions of all players in all preceding slots [1]. As in a one-shot game (see Section 3.2), a Nash equilibrium (NE) in a repeated game is a strategy profile in which no player can improve his payoff by unilateral deviation from his strategy [1]. However, NE constitutes a rather weak notion of equilibria in repeated games [1] and hence we focus on NE with a special property, known as the Subgame Perfect Nash Equilibria (SPNE) [1]. A subgame [1] of the repeated game is the part of the game starting from some slot $\tau_{0} \geq 1$, i.e. the stage games in slots $\tau=\tau_{0}, \tau_{0}+1, \ldots$. An SPNE is an NE of the repeated game that is also an NE of every subgame [1].

\subsection{Results}

It is well-known that for any repeated game, the strategy profile that uses the one shot game NE every time, is a SPNE [1]. Thus, the symmetric NE we presented for the one-shot game in Section 3 provides a SPNE in the repeated game version. The efficiency (as defined in the first paragraph of Section 3.4) of this SPNE is however low whenever the symmetric NE has low efficiency, which happens for certain ranges of $n, k, q$ (Lemma 2). Our main contribution is to present an SPNE that is also efficient in the sense that the sum of expected utilities of the $n$ primaries at equilibrium equals the maximum possible sum of utilities, provided the discount factor $\delta$ is sufficiently high.

We consider Nash reversion type of strategy profiles [1] in which each player plays a specified strategy (called the pre-deviation strategy) at each time until one of the players deviates from it, and all players play the one-shot game NE strategy thereafter.

Strategy for primary i: Select a price of $v$ at $\tau=1$, and also for all other $\tau$ so long as all other primaries had chosen $v$ in all previous times. Otherwise, play the one-shot game Nash equilibrium strategy $\psi($.$) in (6).$ THEOREM 6. The above Nash reversion strategy is an SPNE if $\delta \geq \delta_{t}$, where $\delta_{t}$ is a threshold given by:

$$
\delta_{t}=\frac{w(q, n)-\beta(q, n)}{w(q, n)-\beta(q, n)+q \beta(q, n)}
$$

and

$$
\beta(q, n)=\sum_{i=k}^{n-1}\left(\frac{k}{i+1}\right)\left(\begin{array}{c}
n-1 \\
i
\end{array}\right) q^{i}(1-q)^{n-1-i}
$$

Note that from (2) and (12), $w(q, n)>\beta(q, n)>0$ and hence $0<\delta_{t}<1$. Thus, for all values of $n, k$ and $q$, there exists a threshold such that for values of $\delta$ greater than it, the above Nash reversion strategy is an SPNE.

The efficiency of the above SPNE is 1 because bandwidth is always sold at the highest possible price $v$. Thus, an efficient NE can be sustained in the repeated game, unlike in the one-shot game (Lemma 2). This is possible because of the threat mechanism inherent in the above SPNE: if a primary tries to undercut the prices of other primaries, then he will gain temporarily, but will suffer in the long run because all primaries will switch to the one-shot game NE strategy immediately afterwards.
We plot $\delta_{t}$ versus $q$ in Fig. 5 for different values of $n$. The plot reveals that $\delta_{t}$ is not close to 1 except when $q$ is close to 0 . Thus, since players usually have discount factors $\delta$ close to 1 [1], their discount factors would exceed $\delta_{t}$ except for very small $q$, and hence the above strategy profile will constitute a SPNE unless $q$ is very small. The availability probability of unused bandwidth is rarely close to 0 , and even when it is, we have an alternate SPNE strategy profile whose efficiency is very close to 1 . Now, when $q$ is very small, the lower limit $\tilde{p}$ in (3), of the symmetric NE price distribution $\psi($.$) in the one shot game in$ Section 3 is close to the upper limit $v$ (refer to (6), (5), (4)), and hence the SPNE that uses this distribution for each player at each time provides prices close to $v$ as well at each time, and thereby attains efficiency close to 1 .

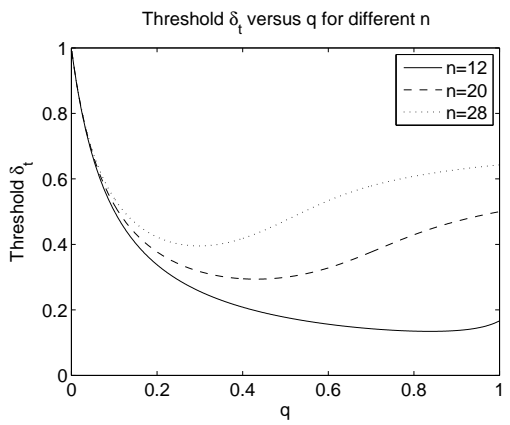

Figure 5. The threshold $\delta_{t}$ versus $q$ for three values of $n$. The other parameters are $c=0, v=100$ and $k=10$.

\section{Generalizations}

We now generalize the models to allow for asymmetric $q$ values of the primaries (Section 6.1), and random valuations of the secondaries (Section 6.2).

\subsection{Asymmetric $q$}

So far, we have assumed that each primary has unused bandwidth with equal probability, $q$. Now, we consider that this probability is $q_{i}$ for primary $i$, and allow for potentially unequal $q_{i} \mathrm{~s}$. This generality leads to some remarkable differences in the NE strategies, which we elucidate considering a simple scenario, $n=2$ and $k=1$. Without loss of generality, let $q_{1} \geq q_{2}$.

We first describe the equilibrium strategies $\psi_{1}($.$) and$ $\psi_{2}$ (.) of the two primaries for the one shot game at a single location. Define:

$$
\tilde{p}_{i}=v-q_{i}(v-c), i=\{1,2\}
$$

Then $\tilde{p}_{1} \leq \tilde{p}_{2}$. Let $\psi_{1}(x)$ be as in (7) with $\tilde{p}_{2}$ in place of $\tilde{p}$ and $q_{1}$ in place of $q$. Also, let $\psi_{2}(x)$ be as in (7) with $\tilde{p}_{2}$ in place of $\tilde{p}$ and $q_{2}$ in place of $q$.

THEOREM 7. The strategy profile in which primary i selects his price using the d.f. $\psi_{i}(),. i=1,2$ is a $N E$

Note that this NE is not in general symmetric, as $\psi_{1}(.) \neq \psi_{2}($.$) , which is but expected as q_{1}, q_{2}$ need not be equal. Also, it can be checked that $\psi_{2}($.$) is continu-$ ous, whereas $\psi_{1}($.$) is not (unless q_{1}=q_{2}$ ), and primary 1 chooses price $v$ with a positive probability $1-\frac{q_{2}}{q_{1}}$. This is in contrast to the NE for equal $q_{i}$ s (Theorem 2), where 
each primary uses a continuous d.f. and hence does not choose any single price with positive probability.

Now, the above results extend to the multiple location case similar to Section 4. Specifically, for the linear graph in part (a) of Fig. 3, the NE strategies are as follows:

1. when $m$ is even, both primaries randomize between $I_{o}$ and $I_{e}$ w.p. $\frac{1}{2}$ each, and at each node in the chosen independent set, primary $i, i \in\{1,2\}$, chooses the price from the distribution $\psi_{i}($.$) above with \frac{q_{j}}{2}$ in place of $q_{j}, j \in\{1,2\}$.

When $m$ is odd:

2. if $q_{2} \leq \frac{2}{m+1}$, each primary $i$ offers bandwidth only at nodes in $I_{o}$, and sets the price at each node according to the d.f. $\psi_{i}($.$) above.$

3. if $q_{2}>\frac{2}{m+1}$, each primary offers bandwidth at $I_{e}$ (resp., $I_{o}$ ) w.p. $t$ (resp., $1-t$ ), where $t$ is the solution of (11) with $q_{2}$ in place of $q$. Also, at each node in $I_{e}$ (resp., $I_{o}$ ), primary $i, i \in\{1,2\}$, chooses the price from the distribution $\psi_{i}($.$) above with t q_{j}$ (resp., $\left.(1-t) q_{j}\right), j \in\{1,2\}$ in place of $q$.

The NE for the grid graph are similar, with the changes mentioned in Section 4.2.

\subsection{Random Valuations}

Recall that in Section 3.1, we noted that the parameter $v$ may also be interpreted as the valuation of each buyer. Then the primaries may only know a distribution, but not the exact value, of $v$. We now generalize the results in the single location model in Section 3, which were for a deterministic $v$, to the case where $v$ is distributed according to the d.f. $G(x)=P(v \leq x)$. Assume that $G(x)$ is continuous. Also, let $g(x)=(x-c) P(v \geq x)$. Assume, in addition, that: (i) $g(x)$ has a unique maximizer $v_{T}>c$, and (ii) $g(x)$ is strictly increasing for $c \leq x \leq v_{T}$. Note that a large class of d.f. $G(x)$ satisfy the above technical conditions, e.g., the uniform distribution on some range $[\underline{v}, \bar{v}]$, where $c<\underline{v}<\bar{v}$. Note that by continuity of $G(x)$, $g(x)=(x-c)(1-G(x))$ is continuous. For analytical tractability, we restrict ourselves to the case $k=1 ; n$ can be arbitrary.

First, note that if there is only one primary, then he sells his unused bandwidth whenever his price does not exceed the secondary's (random) valuation. Thus, his expected utility is $g(p)=(p-c) P(v \geq p)$ when he quotes a price $p \geq c$ for his unused bandwidth. The optimal price that maximizes this expected payoff is $v_{T}$, the maximizer of $g(x)$.

Now, suppose there are $n$ primaries and each primary $i$ chooses the price $p_{i} \in\left[c, v_{T}\right]$ according to the d.f. $\psi($.$) .$ As in the constant valuation case in Section 3.3, $\phi($.$) is the$ distribution of a pseudo-price and $F(x)$ is the distribution of the minimum of $(n-1)$ pseudo-prices. A primary's pseudo-price is greater than $v_{T}$ if he has no unused bandwidth. So:

$$
1-F\left(v_{T}\right)=(1-q)^{n-1}
$$

Hence:

$$
F\left(v_{T}\right)=1-(1-q)^{n-1}
$$

Now, if primary $i$ sets a price $p_{i}=x$, then he sells his bandwidth if the minimum of the pseudo-prices of the primaries other than $i$ is greater than $x$ and $v \geq x$. So the expected utility of primary $i$ if he sets a price $p_{i}=x$ and all other primaries choose the price according to the d.f. $\psi($.$) is:$

$$
E\left\{u_{i}\left(x, \psi_{-i}\right)\right\}=(x-c)(1-F(x)) P(v \geq x)
$$

In a NE, this should be a constant over the range $\left[\tilde{p}, v_{T}\right]$ for some $c<\tilde{p}<v_{T}$ and must equal:

$$
\begin{aligned}
E\left\{u_{i}\left(\tilde{p}, \psi_{-i}\right)\right\} & =(\tilde{p}-c)(1-F(\tilde{p})) P(v \geq \tilde{p}) \\
& =(\tilde{p}-c) P(v \geq \tilde{p})
\end{aligned}
$$

since $F(\tilde{p})=0$. By (14) and (15):

$(x-c)(1-F(x)) P(v \geq x)=(\tilde{p}-c) P(v \geq \tilde{p}), x \in\left[\tilde{p}, v_{T}\right]$

Thus,

$$
F(x)=1-\frac{(\tilde{p}-c) P(v \geq \tilde{p})}{(x-c) P(v \geq x)}, x \in\left[\tilde{p}, v_{T}\right]
$$

Note that since $g(x)=(x-c) P(v \geq x)$ is increasing, $0 \leq$ $F(x) \leq 1$ and $F(x)$ is increasing. Now, $\tilde{p}$ can be found from (13) and (16) to be the solution of:

$$
\frac{(\tilde{p}-c) P(v \geq \tilde{p})}{\left(v_{T}-c\right) P\left(v \geq v_{T}\right)}=(1-q)^{n-1}
$$

Now, $\phi(x)$ is the d.f. such that the minimum of $(n-1)$ i.i.d. random variables, each with d.f. $\phi($.$) , has the d.f.$ $F($.). So similar to (5):

$$
F(x)=1-(1-\phi(x))^{n-1}
$$

Equations (16) and (18) provide an expression for $\phi($.$) .$ Also, similar to Theorem 2, we have:

THEOREM 8. The strategy profile in which each primary plays $\psi($.$) , where$

$$
\psi(x)= \begin{cases}0, & x \leq \tilde{p} \\ \frac{1}{q} \phi(x), & \tilde{p}<x \leq v_{T} \\ 1, & x>v_{T}\end{cases}
$$

and $\phi($.$) is defined by (16) and (18) is a symmetric NE.$ The proof is similar to that of Theorem 2 .

\subsubsection{Uniformly Distributed Valuation}

Now, we specialize our results to the case in which $v$ is uniformly distributed in $[\underline{v}, \bar{v}]$, where $c<\underline{v}<\bar{v}$, and explicitly compute $F(x)$, which then can be used to compute $\phi(x)$ and $\psi(x)$. Since $v$ is uniformly distributed in $[\underline{v}, \bar{v}]$, it can be checked that $v_{T}=\max \left\{\underline{v}, \frac{\bar{v}+c}{2}\right\}$. If $\underline{v} \geq \frac{\bar{v}+c}{2}$, then $v_{T}=\underline{v}$ and the results in the constant valuation case go through with $v$ replaced by $\underline{v}$. This is because for every primary $i$, any price $p_{i}>\underline{v}$ fetches an expected utility which is lower than that for $p_{i}=\underline{v}$. Thus, henceforth, we consider $v_{T}=\frac{\bar{v}+c}{2}$.

LEMMA 5. There exists a unique $\tilde{p}_{1}$ in $\left(c, v_{T}\right)$ such that:

$$
\left(\tilde{p}_{1}-c\right)\left(\bar{v}-\tilde{p}_{1}\right)=\frac{(1-q)^{n-1}(\bar{v}-c)^{2}}{4} \text {. }
$$

We consider the cases $\tilde{p}_{1} \geq \underline{v}$ and $\tilde{p}_{1}<\underline{v}$ separately. 


\subsubsection{Case I: $\tilde{p}_{1} \geq \underline{v}$}

In this case, it can be checked that the $\tilde{p}$ in (17) is equal to $\tilde{p}_{1}$. Also, $F(x)$ in (16) becomes:

$$
F(x)= \begin{cases}0, & x \leq \tilde{p}_{1} \\ 1-\frac{\left(\tilde{p}_{1}-c\right)\left(\bar{v}-\tilde{p}_{1}\right)}{(x-c)(\bar{v}-x)}, & \tilde{p}_{1}<x \leq v_{T}\end{cases}
$$

\subsubsection{Case II: $\tilde{p}_{1}<\underline{v}$}

Lemma 6. There exists a unique $\tilde{p}_{2}$ in $\left(\tilde{p}_{1}, \underline{v}\right)$ such that:

$$
\frac{4\left(\tilde{p}_{2}-c\right)(\bar{v}-\underline{v})}{(\bar{v}-c)^{2}}=(1-q)^{n-1} .
$$

In this case, it can be checked that the $\tilde{p}$ in (17) is equal to $\tilde{p}_{2}$. Also, $F(x)$ in (16) becomes:

$$
F(x)= \begin{cases}0, & x \leq \tilde{p}_{2} \\ \frac{x-\tilde{p}_{2}}{x-c}, & \tilde{p}_{2}<x \leq \underline{v} \\ 1-\frac{\left(\tilde{p}_{2}-c\right)(\bar{v}-\underline{v})}{(x-c)(\bar{v}-x)}, & \underline{v}<x \leq v_{T}\end{cases}
$$

\section{Conclusions and Future Work}

We analyzed price competition in a CRN with multiple primaries and secondaries, taking into account bandwidth uncertainty and spatial reuse. We computed NE in linear and grid conflict graphs; an important problem for future research is the extension of these results to general conflict graphs. Also, we assumed that a secondary buys bandwidth if and only if the price is less than or equal to his valuation. This is an optimal strategy for a secondary in a one-shot game. However, for a repeated game, it may not be optimal-for example a secondary may choose not to buy bandwidth in a slot even if the price is less than his valuation, so as to induce primaries to lower prices in future slots. The analysis of price competition in the presence of such strategic behavior by secondaries is an interesting problem for future work. Finally, extension of the results in this paper to the case where each primary owns multiple bands is another direction for future research.

\section{Acknowledgments}

The contributions of both authors have been supported by NSF grants $0621782,0721308,0914955,0915203$, 0915697.

\section{References}

[1] A. Mas-Colell, M. Whinston, J. Green, "Microeconomic Theory”, Oxford University Press, 1995.

[2] I. Akyildiz, W.-Y. Lee, M. Vuran, S. Mohanty "NeXt generation/dynamic spectrum access/cognitive radio wireless networks: a survey". In Comp. Networks, Vol. 50, 13, pp. 2127-59, 2006.

[3] S.-F. Cheng, D.M. Reeves, Y. Vorobeychik, M.P. Wellman, "Notes on Equilibria in Symmetric Games", In AAMAS-04 Workshop on Game-Theoretic and Decision-Theoretic Agents, 2004.

[4] M. J. Osborne, and C. Pitchik, "Price Competition in a Capacity-Constrained Duopoly", In Journal of Economic Theory, 38(2), pp. 238-260, 1986.
[5] D.M. Kreps, J.A. Scheinkman, "Quantity Precommitment and Bertrand Competition Yield Cournot Outcomes", In Bell Journal of Economics, 14, pp. 326-337, Autumn 1983.

[6] M. Janssen, E. Rasmusen "Bertrand Competition Under Uncertainty", In Journal of Industrial Economics, 50(1): pp. 11-21, March 2002.

[7] C. Courcoubetis and R. Weber, "Pricing Communication Networks", John Wiley \& Sons, Ltd. 2003.

[8] J.E. Harrington, "A Re-Evaluation of Perfect Competition as the Solution to the Bertrand Price Game”, In Math. Soc. Sci. , 17, pp. 315-328, 1989.

[9] J. E. Walsh, "Existence of Every Possible Distribution for any Sample Order Statistic", In Statistical Papers, Vol. 10, No. 3, Springer Berlin, Sept. 1969.

[10] H.R. Varian, "A Model of Sales", In American Economic Review, 70, pp. 651-659, 1980.

[11] B. Hajek, G. Sasaki "Link Scheduling in Polynomial Time", In IEEE Trans. on Information Theory, Vol. 34, No. 5, Sept. 1988.

[12] W. Rudin, "Principles of Mathematical Analysis", Mc-Graw Hill, Third Edition, 1976.

[13] H.A. David, H.N. Nagaraja, "Order Statistics”, Wiley, New Jersey, Third Edition, 2003.

[14] S. Ross, "Stochastic Processes", Wiley, Second Edition, 1995.

[15] R. Myerson, "Game Theory: Analysis of Conflict", Harvard University Press, 1997.

[16] O. Ileri, D. Samardzija, T. Sizer, N. B. Mandayam, "Demand Responsive Pricing and Competitive Spectrum Allocation via a Spectrum Policy Server", In Proc. of IEEE DySpan, 2005.

[17] P. Maille, B. Tuffin "Analysis of Price Competition in a Slotted Resource Allocation Game", In Proc. of Infocom, 2008.

[18] P. Maille, B. Tuffin, "Price War with Partial Spectrum Sharing for Competitive Wireless Service Providers", In Proc. of IEEE Globecom, Dec. 2009.

[19] Y. Xing, R. Chandramouli, C. Cordeiro, "Price Dynamics in Competitive Agile Spectrum Access Markets", In IEEE JSAC, Vol. 25, No. 3, April 2007.

[20] D. Niyato, E. Hossain, "Competitive Pricing for Spectrum Sharing in Cognitive Radio Networks: Dynamic Game, Inefficiency of Nash Equilibrium, and Collusion", IEEE JSAC, Vol. 26, No. 1, 2008.

[21] D. Niyato, E. Hossain, Z. Han, "Dynamics of Multiple-Seller and Multiple-Buyer Spectrum Trading in Cognitive Radio Networks: A GameTheoretic Modeling Approach", IEEE TMC, Vol. 8, No. 8, pp. 1009-1022, Aug. 2009.

[22] X. Zhou, H. Zheng, "TRUST: A General Framework for Truthful Double Spectrum Auctions", In 
Proc. of Infocom, April 2009.

[23] D. West, Introduction to Graph Theory, 2nd ed., Prentice Hall, 2000.

[24] B.S. Everitt, The Cambridge Dictionary of Statistics, 3rd ed., Cambridge University Press, 2006.

[25] S. Chawla and T. Roughgarden, "Bertrand Competition in Networks", In Symposium on Algorithmic Game Theory, May, 2008.

[26] W. Hoeffding, "Probability inequalities for sums of bounded random variables", Journal of the American Statistical Association 58 (301): 1330, March 1963.

\section{Appendix}

\section{A Proof of Theorem 1}

Before proving Theorem 1, we state a definition. A strategy $p_{i}$ of player $i$ is said to strictly dominate [1] another strategy $p_{i}^{\prime}$ if:

$$
E\left\{u_{i}\left(p_{i}, p_{-i}\right)\right\}>E\left\{u_{i}\left(p_{i}^{\prime}, p_{-i}\right)\right\}, \forall p_{-i}
$$

Proof of THEOREM 1 . For every primary $i$, and any $p_{-i}, u_{i}\left(c, p_{-i}\right)=0$. Also, $E\left\{u_{i}\left(p_{i}, p_{-i}\right)\right\}>0$ for all $p_{i} \in(c, v]$ because primary $i$ gets a positive payoff in the event that no other primary has unused bandwidth, which happens with positive probability. Thus, the strategy $p_{i}=c$ is strictly dominated by each $p_{i} \in(c, v]$, and hence no primary sets $p_{i}=c$ in any pure-strategy Nash equilibrium.

Suppose $\left(p_{1}, \ldots, p_{n}\right)$ is a pure-strategy Nash equilibrium, where $c<p_{i} \leq v$ for $i=1, \ldots, n$. Let $p_{\min }=$ $\min \left(p_{1}, \ldots, p_{n}\right), S_{\text {min }}=\left\{i: p_{i}=p_{\min }\right\}$, and $n_{\text {min }}=\left|S_{\text {min }}\right|$. Note that $S_{\min }$ is the set of primaries who set the lowest price $p_{\min }$, and $n_{\min }$ is its cardinality. One of the following two cases must hold:

Case (i): $n_{\min } \leq k$

Since $k \leq n-1, n_{\min } \leq n-1$ and hence at least one primary sets a price above $p_{\min }$. Since $p_{i} \leq v, i=1, \ldots, n$, it follows that $p_{\min }<v$.

Let $p_{j}=\min \left\{p_{i}: i \notin S_{\min }\right\}$ be the second lowest price. Now, note that $\forall i \in S_{\text {min }}, u_{i}\left(p_{\min }, p_{-i}\right)=p_{\text {min }}-c$ and $u_{i}\left(p_{i}^{\prime}, p_{-i}\right)=p_{i}^{\prime}-c \forall p_{i}^{\prime} \in\left(p_{\min }, p_{j}\right)$. This is because the bandwidth of primary $i$ always gets sold for any $p_{i}^{\prime}<p_{j}$, since it is among the primaries with the $n_{\min } \leq k$ lowest prices. So $\forall i \in S_{\min }$ :

$$
u_{i}\left(p_{\min }, p_{-i}\right)<u_{i}\left(p_{i}^{\prime}, p_{-i}\right) \forall p_{i}^{\prime} \in\left(p_{\min }, p_{j}\right)
$$

Hence $p_{i}=p_{\min }$ is not a best response to $p_{-i}$, which contradicts the assumption that $\left(p_{1}, \ldots, p_{n}\right)$ is a Nash equilibrium.

Case (ii): $n_{\min }>k$

In this case, for $i \in S_{\min }$ :

$$
E\left\{u_{i}\left(p_{\text {min }}, p_{-i}\right)\right\}=\left(p_{\text {min }}-c\right) P\left(E_{1}\right)
$$

where $E_{1}$ is the event that primary $i$ 's bandwidth is bought by a secondary. Note that $P\left(E_{1}\right)<1$ because with a positive probability, $k$ or more primaries, other than $i$, in $S_{\text {min }}$ have unused bandwidth. In this case, $k$ randomly selected primaries, out of the primaries in $S_{\min }$ who have unused bandwidth, sell their bandwidth, and with a positive probability, primary $i$ is not among them. Also, note that primary $i$ 's bandwidth is always sold if it sets a price less than $p_{\min }$ and the vector of prices of primaries other than $i$ is $p_{-i}$. Hence, for small enough $\varepsilon>0$ :

$$
\begin{aligned}
E\left\{u_{i}\left(p_{\text {min }}-\varepsilon, p_{-i}\right)\right\} & =\left(p_{\text {min }}-\varepsilon-c\right) \\
& >\left(p_{\min }-c\right) P\left(E_{1}\right) \\
& =E\left\{u_{i}\left(p_{\text {min }}, p_{-i}\right)\right\}
\end{aligned}
$$

Thus, $p_{i}=p_{\min }$ is not a best response, which contradicts the assumption that $\left(p_{1}, \ldots, p_{n}\right)$ is a Nash equilibrium.

\section{B Proof of Theorem 2 \\ B.1 Proof of Lemma 1}

Proof of Lemma 1. Let

$$
\mathcal{F}(y)=\sum_{i=k}^{n-1}\left(\begin{array}{c}
n-1 \\
i
\end{array}\right) y^{i}(1-y)^{n-1-i}, y \in[0,1]
$$

$\mathcal{F}(y)$ is a continuous and strictly increasing function and $\mathcal{F}(0)=0, \mathcal{F}(1)=1$ [9]. So $\mathcal{F}($.$) is invertible. Since$ $F(x)=\mathcal{F}(\phi(x))$, we get that $\phi($.$) is unique and given by:$

$$
\phi(x)=\mathcal{F}^{-1}(F(x))
$$

Also, since $\mathcal{F}$ is a continuous one-to-one map from the compact set $[0,1]$ onto $[0,1], \mathcal{F}^{-1}$ is continuous (see Theorem 4.17 in [12]). Also, $F(x)$ is continuous. So from (23), $\phi(x)$ is a continuous function of $x$, since it is the composition of continuous functions $\mathcal{F}^{-1}$ and $F$ (see Theorem 4.7 in [12]). Now, $F(x)=\mathcal{F}(\phi(x)), F(\tilde{p})=0$ and $F(v)=w(q, n)$. So $0=\mathcal{F}(\phi(\tilde{p}))$ and $w(q, n)=$ $\mathcal{F}(\phi(v))$. But $\phi(\tilde{p})=0$ and $\phi(v)=q$ satisfy the above equations, by (2). The result follows.

\section{B.2 Proof that (6), (5), and (4) Constitute an NE Strategy}

First, we show that the strategy profile in which each primary $i$ plays the strategy $\psi($.$) defined by (6), (5), and$ (4) is a NE. Suppose primaries $\{1, \ldots, n\} \backslash i$ play the strategy $\psi($.$) . Recall from Section 3.3$ that $p_{(k)}^{\prime}$ is the $k^{\prime}$ th smallest among the pseudo-prices of primaries other than primary $i$, and $F(x)=P\left(p_{(k)}^{\prime} \leq x\right)$. Also, if primary $i$ selects a price $p_{i}=x$, then its payoff is $(x-c)$ if $p_{(k)}^{\prime}>x$ and 0 if $p_{(k)}^{\prime}<x$. Since $F(x)$ in (4) is continuous for $x \in[\tilde{p}, v], P\left(p_{(k)}^{\prime}=x\right)=0$ for $x \in[\tilde{p}, v]$. So primary $i$ 's expected payoff for each price $x \in[\tilde{p}, v]$ is given by:

$$
\begin{aligned}
E\left\{u_{i}\left(x, \Psi_{-i}\right)\right\} & =(x-c) P\left(p_{(k)}^{\prime}>x\right) \\
& =(x-c)(1-F(x)) \\
& =(x-c)\left\{1-\left(\frac{x-\tilde{p}}{x-c}\right)\right\} \\
& =\tilde{p}-c
\end{aligned}
$$

Also, primary $i$ 's expected payoff for a price $p_{i}<\tilde{p}$ is $p_{i}-c<\tilde{p}-c$ and that for a price $p_{i}>v$ is 0 . So each $p_{i} \in$ $[\tilde{p}, v]$ is a best response. Since $\psi($.$) randomizes among$ 
the prices in $[\tilde{p}, v], \psi($.$) is a best response for primary i$. Hence, the strategy profile in which each primary $i$ plays $\psi($.$) is an NE.$

\section{B.3 Uniqueness}

Suppose in a symmetric NE, primaries $1, \ldots, n$ set their prices according to some common distribution $\psi(x)$. Let $\phi(x)$ be the d.f. of each pseudo-price, $p_{i}^{\prime}, i=1, \ldots, n$ and $F(x)$ be the d.f. of $p_{(k)}^{\prime}$, which is the $k^{\prime}$ th smallest of $p_{2}^{\prime}, \ldots, p_{n}^{\prime}$, as described in Section 3.3. We will show that $\psi(x), \phi(x)$ and $F(x)$ satisfy (6), (5), (4).

LEMMA 7. $F(x)$ is continuous on $x \in[c, v]$.

ProOF. Suppose $F(x)$ is not continuous on $[c, v]$. Then it has a jump, say at $x_{0} \in[c, v]$, i.e. $P\left\{p_{(k)}^{\prime}=x_{0}\right\}>0$. As shown in the proof of Theorem 1 , the strategy $p_{i}=c$ is strictly dominated for each primary $i$. Hence, primary $i$ plays $p_{i}=c$ with 0 probability; so $\psi($.$) , and thereby F($.$) ,$ has no jump at $c$. Thus, $x_{0}>c$. We will show that primary 1 gets a higher utility by setting $p_{1}$ slightly lower than $x_{0}$, than by setting $p_{1}=x_{0}$, which contradicts the fact that $x_{0}$ is a best response. Let $E_{2}$ denote the event that primary 1 's bandwidth is sold. We have:

$$
\begin{array}{r}
P\left\{E_{2} \mid p_{1}=x_{0}\right\}=P\left\{p_{(k)}^{\prime}>x_{0}\right\} \\
+P\left\{E_{2} \mid p_{1}=x_{0}, p_{(k)}^{\prime}=x_{0}\right\} P\left\{p_{(k)}^{\prime}=x_{0}\right\}
\end{array}
$$

and for every $\varepsilon>0$ :

$$
P\left\{E_{2} \mid p_{1}=x_{0}-\varepsilon\right\} \geq P\left\{p_{(k)}^{\prime}>x_{0}\right\}+P\left\{p_{(k)}^{\prime}=x_{0}\right\}
$$

By (25) and (26):

$$
\begin{array}{r}
P\left\{E_{2} \mid p_{1}=x_{0}-\varepsilon\right\}-P\left\{E_{2} \mid p_{1}=x_{0}\right\} \geq \\
P\left\{p_{(k)}^{\prime}=x_{0}\right\}\left(1-P\left\{E_{2} \mid p_{1}=x_{0}, p_{(k)}^{\prime}=x_{0}\right\}\right)=a \text { (say) }
\end{array}
$$

Note that $P\left\{E_{2} \mid p_{1}=x_{0}, p_{(k)}^{\prime}=x_{0}\right\}<1$ and hence $a>0$.

Now, primary 1's expected utility if he sets $p_{1}=x_{0}$ is:

$$
E\left\{u_{1}\left(x_{0}, \psi_{-1}\right)\right\}=\left(x_{0}-c\right) P\left\{E_{2} \mid p_{1}=x_{0}\right\}
$$

and if he sets $x_{0}-\varepsilon$ for a small $\varepsilon>0$ is:

$$
\begin{aligned}
E\left\{u_{1}\left(x_{0}-\varepsilon, \psi_{-1}\right)\right\} & =\left(x_{0}-\varepsilon-c\right) P\left\{E_{2} \mid p_{1}=x_{0}-\varepsilon\right\} \\
& \geq\left(x_{0}-\varepsilon-c\right)\left(P\left\{E_{2} \mid p_{1}=x_{0}\right\}+a\right)
\end{aligned}
$$

by (27). Taking limits:

$$
\begin{aligned}
\lim _{\varepsilon \rightarrow 0+} E\left\{u_{1}\left(x_{0}-\varepsilon, \psi_{-1}\right)\right\} & \geq\left(x_{0}-c\right)\left(P\left\{E_{2} \mid p_{1}=x_{0}\right\}+a\right) \\
& >E\left\{u_{1}\left(x_{0}, \psi_{-1}\right)\right\}
\end{aligned}
$$

Thus, for small enough $\varepsilon, p_{1}=x_{0}-\varepsilon$ yields a higher expected payoff than $p_{1}=x_{0}$. So $p_{1}=x_{0}$ is not a best response, which is a contradiction. Thus, $F(x)$ cannot have a jump and is continuous.

By continuity of $F($.$) , for any x, P\left(p_{(k)}^{\prime}=x\right)=0$ and hence:

$$
E\left\{u_{1}\left(x, \psi_{-1}\right)\right\}=(x-c)(1-F(x))
$$

Next, we show that the set of best responses of primary 1 to the vector of strategies $\psi_{-1}$, in which each of the primaries $2, \ldots, n$ play $\psi($.$) , is a contiguous set.$
LEMMA 8. The set of best responses of primary 1 is convex.

PROOF. Let $z_{l}$ and $z_{r}$ be best responses, where $c<z_{l}<$ $z_{r} \leq v$. We show that every $z \in\left(z_{l}, z_{r}\right)$ is a best response. If not, then suppose $z_{0} \in\left(z_{l}, z_{r}\right)$ is not a best response. One of the following cases must hold:

Case $(i)$ : For every $\varepsilon>0$, there exists a $z \in\left(z_{0}-\varepsilon, z_{0}+\varepsilon\right)$ that is a best response. Then we can find a sequence $z_{1}, z_{2}, z_{3}, \ldots$ such that each $z_{i}, i=1,2,3, \ldots$ is a best response and $\lim _{i \rightarrow \infty} z_{i}=z_{0}$ [12]. By (28):

$$
\begin{aligned}
E\left\{u_{1}\left(z_{0}, \psi_{-1}\right)\right\}= & \left(z_{0}-c\right)\left(1-F\left(z_{0}\right)\right) \\
= & \lim _{i \rightarrow \infty}\left(z_{i}-c\right)\left(1-F\left(z_{i}\right)\right) \\
& (\text { by continuity of } F(.)) \\
= & \left(z_{1}-c\right)\left(1-F\left(z_{1}\right)\right)
\end{aligned}
$$

since $\left(z_{i}-c\right)\left(1-F\left(z_{i}\right)\right)$ is equal for $i=1,2,3, \ldots$ and equals the maximum expected payoff. Thus, $p_{1}=z_{0}$ yields the maximum expected payoff and $z_{0}$ is a best response, which is a contradiction.

Case (ii): There exists an interval $\left(z_{0}-\varepsilon, z_{0}+\varepsilon\right)$ such that no $z$ in that interval is a best response. Let:

$$
\begin{aligned}
& \tilde{z}_{l}=\sup \left\{z \leq z_{0}-\varepsilon: z \text { is a best response }\right\} \\
& \tilde{z}_{r}=\inf \left\{z \geq z_{0}+\varepsilon: z \text { is a best response }\right\}
\end{aligned}
$$

Note that the supremum and infimum exist because $z_{l}$ and $z_{r}$ are best responses and hence the sets over which the supremum and infimum are taken are non-empty. Now, similar to (29), it can be shown using continuity of $F($. that $\tilde{z}_{l}$ and $\tilde{z}_{r}$ are best responses. So:

$$
E\left\{u_{1}\left(\tilde{z}_{l}, \psi_{-1}\right)\right\}=E\left\{u_{1}\left(\tilde{z}_{r}, \psi_{-1}\right)\right\}=u_{\max }
$$

where $u_{\max }$ is the maximum expected utility. By (28):

$$
\begin{aligned}
& F\left(\tilde{z}_{l}\right)=1-\frac{u_{\max }}{\tilde{z}_{l}-c} \\
& F\left(\tilde{z}_{r}\right)=1-\frac{u_{\max }}{\tilde{z}_{r}-c}
\end{aligned}
$$

By the above two equations, since $u_{\max }>0$ and $\tilde{z}_{l}<\tilde{z}_{r}$ :

$$
F\left(\tilde{z}_{l}\right)<F\left(\tilde{z}_{r}\right)
$$

But since no $z \in\left(\tilde{z}_{l}, \tilde{z}_{r}\right)$ is a best response, for every primary $i, P\left(p_{i} \in\left(\tilde{z}_{l}, \tilde{z}_{r}\right)\right)=0$. Hence, $P\left(p_{(k)}^{\prime} \in\left(\tilde{z}_{l}, \tilde{z}_{r}\right)\right)=0$.

That is, $F\left(\tilde{z}_{r}-\right)-F\left(\tilde{z}_{l}\right)=0$, where $F(x-)=\lim _{y \uparrow x} F(y)$. By continuity of $F(),. F\left(\tilde{z}_{r}\right)=F\left(\tilde{z}_{r}-\right)$. So $F\left(\tilde{z}_{l}\right)=F\left(\tilde{z}_{r}\right)$, which contradicts (30).

Proof of Uniqueness In Theorem 2. By

Lemma 8, the set of best responses of primary 1 is convex, and hence is an interval. Also, by continuity of $F(x)$, it can be shown similar to (29) that the endpoints of this interval are best responses, i.e. the interval is closed. Let $\left[z_{l}, z_{r}\right]$ be this interval for some $c<z_{l}<z_{r} \leq v$. We now show that $z_{r}=v$ and $z_{l}=\tilde{p}$.

Suppose $z_{r}<v$. As shown in Section 3.3, $F(v)=$ $w(q, n)$. Now, $P\left(p_{i} \in\left(z_{r}, v\right]\right)=0$. So, $P\left(p_{(k)}^{\prime} \in\left(z_{r}, v\right]\right)=0$ and hence:

$$
F\left(z_{r}\right)=F(v)=w(q, n)
$$


By (28):

$$
\begin{aligned}
E\left\{u_{1}\left(z_{r}, \psi_{-1}\right)\right\}= & \left(z_{r}-c\right)\left(1-F\left(z_{r}\right)\right) \\
= & \left(z_{r}-c\right)(1-w(q, n)) \\
& (\text { by }(31))
\end{aligned}
$$

and

$$
\begin{aligned}
E\left\{u_{1}\left(v, \psi_{-1}\right)\right\} & =(v-c)(1-F(v)) \\
& =(v-c)(1-w(q, n))(\text { by }(31)) \\
& >E\left\{u_{1}\left(z_{r}, \psi_{-1}\right)\right\}(\text { by }(32))
\end{aligned}
$$

which contradicts the fact that $z_{r}$ is a best response. Hence, we must have $z_{r}=v$.

The maximum payoff is $(v-c)(1-F(v))=(v-$ $c)(1-w(q, n))=\tilde{p}-c$. Now, note that $F\left(z_{l}\right)=0$. Putting $x=z_{l}$ and $F\left(z_{l}\right)=0$ in (28):

$$
E\left\{u_{1}\left(z_{l}, \psi_{-1}\right)\right\}=\left(z_{l}-c\right)(1-0)=\tilde{p}-c
$$

and hence $z_{l}=\tilde{p}$.

Thus, the set of best responses of primary 1 is $[\tilde{p}, v]$.

For any $x \in[\tilde{p}, v], F(x)$ satisfies:

$$
E\left\{u_{1}\left(x, \psi_{-1}\right)\right\}=(x-c)(1-F(x))=\tilde{p}-c
$$

which shows that $F(x)$ is given by (4).

Now, as shown in Section 3.3, $\phi(x)$ must satisfy (5) and by Lemma 1, this $\phi(x)$ is unique. Finally, since $\phi(x)=q \psi(x)$ as shown in Section 3.3, $\psi($.$) is given by$ (6).

\section{Proof of Lemma 2}

Proof of Lemma 2. Since $Z$, the number of primaries who have unused bandwidth, has a Binomial distribution with parameters $n$ and $q$, its mean and variance are $E(Z)=n q$ and $\operatorname{var}(Z)=n q(1-q)$ respectively. First, let $k \leq(n-1)(q-\varepsilon)$ for some $\varepsilon>0$. Let the random variable $Y$ be defined as:

$$
Y= \begin{cases}k, & \text { if } Z \geq k \\ 0, & \text { else }\end{cases}
$$

Then:

$$
\begin{aligned}
& E\{\min (Z, k)\} \\
\geq & E(Y) \\
= & k P(Z \geq k) \\
= & k(1-P(Z<k)) \\
\geq & k(1-P(Z \leq(n-1)(q-\varepsilon))) \\
& (\text { since } k \leq(n-1)(q-\varepsilon)) \\
\geq & k(1-P(|Z-n q| \geq(n-1) \varepsilon)) \\
\geq & k\left(1-\frac{n q(1-q)}{(n-1)^{2} \varepsilon^{2}}\right)
\end{aligned}
$$

(by Chebyshev's inequality [14])

Now, let $Z_{1}$ be a $\operatorname{Binomial}(n-1, q)$ random variable. Note that $E\left(Z_{1}\right)=(n-1) q$ and $\operatorname{var}\left(Z_{1}\right)=(n-1) q(1-$ q). By (2):

$$
\begin{aligned}
1-w(q, n)= & P\left(Z_{1}<k\right) \\
\leq & P\left(Z_{1} \leq(n-1)(q-\varepsilon)\right) \\
& (\text { since } k \leq(n-1)(q-\varepsilon)) \\
\leq & P\left(\left|Z_{1}-(n-1) q\right| \geq(n-1) \varepsilon\right) \\
\leq & 2 \exp \left(\frac{-2(n-1)^{2} \varepsilon^{2}}{n-1}\right)
\end{aligned}
$$$$
\text { (by Hoeffding's inequality [26]) }
$$$$
=2 \exp \left(-2(n-1) \varepsilon^{2}\right)
$$

By (10), (33) and (34):

$$
\eta \leq \frac{2 n q \exp \left(-2(n-1) \varepsilon^{2}\right)}{k\left(1-\frac{n q(1-q)}{(n-1)^{2} \varepsilon^{2}}\right)} \rightarrow 0 \text { as } n \rightarrow \infty
$$

which proves the first part.

Now, suppose $k \geq(n-1)(q+\varepsilon)$ for some $\varepsilon>0$. Since $E\{\min (Z, k)\} \leq E(Z)=n q$, by (10):

$$
\begin{aligned}
\eta \geq & \frac{n q(1-w(q, n))}{n q} \\
= & 1-w(q, n) \\
= & 1-P\left(Z_{1} \geq k\right) \\
\geq & 1-P\left(Z_{1} \geq(n-1)(q+\varepsilon)\right) \\
& (\text { since } k \geq(n-1)(q+\varepsilon)) \\
\geq & 1-P\left(\left|Z_{1}-(n-1) q\right| \geq(n-1) \varepsilon\right) \\
\geq & 1-\frac{(n-1) q(1-q)}{(n-1)^{2} \varepsilon^{2}} \text { (by Chebyshev's inequality) } \\
\rightarrow & 1 \text { as } n \rightarrow \infty
\end{aligned}
$$

which proves the second part.

\section{Proofs of Results in Section 4.1}

We first prove Lemma 4. Let I denote the set of all independent sets in the linear graph with $m$ nodes. In a symmetric NE, suppose each primary $i$ plays independent set $I \in \mathbf{I}$ with probability $\beta(I)$. We will show that $\beta(I)=$ $0, \forall I \neq I_{o}, I_{e}$.

Recall that $\alpha_{j}$ is the total probability with which a primary offers bandwidth at node $j$. Note that:

$$
\alpha_{j}+\alpha_{j+1} \leq 1, j=1, \ldots, m-1
$$

This is because, if $\alpha_{j}+\alpha_{j+1}>1$ for some $j$, then with a positive probability, each primary offers bandwidth at both nodes $j$ and $j+1$. Moreover, we have the following result:

LEMMA 9. If $\alpha_{j}, j=1, \ldots, m$ are probabilities satisfying (35), then there exist probabilities $\beta(I), I \in \mathbf{I}$ such that if a primary plays independent set I with probability $\beta(I)$, then the total probability with which it offers bandwidth at node $j$ is $\alpha_{j}$.

PROOF. We prove this result by a reduction to a scheduling problem and using a result in [11]. From the linear graph $\mathcal{G}_{m}$ with nodes $1, \ldots, m$, construct a linear graph $\mathcal{G}_{m+1}^{\prime}$ with nodes $a_{0}, a_{1}, \ldots, a_{m}$ and edges $\left(a_{j-1}, a_{j}\right), j=$ 
$1,2, \ldots, m$. For $j=1,2, \ldots, m$, let node $j$ in graph $\mathcal{G}_{m}$ correspond to edge $\left(a_{j-1}, a_{j}\right)$ in graph $\mathcal{G}_{m+1}^{\prime}$.

Recall that a matching in a graph is a subset of the edges such that no two edges in the subset have a node in common [23]. Note that a set of nodes $I$ constitutes an independent set in graph $\mathcal{G}_{m}$ if and only if the corresponding set of edges constitutes a matching in graph $\mathcal{G}_{m+1}^{\prime}$. Let $\mathbf{M}$ be the set of all matchings in graph $\mathcal{G}_{m+1}^{\prime}$ and $M(I) \in \mathbf{M}$ be the matching in graph $\mathcal{G}_{m+1}^{\prime}$ corresponding to the independent set $I$ in graph $\mathcal{G}_{m}$.

It has been shown in [11] (see the corollary to Lemma 1 in [11]) that if $\alpha_{j}, j=1, \ldots, m$ are given numbers in $[0,1]$ satisfying $(35)$, then there exists a probability distribution $\left\{\beta^{\prime}(M): M \in \mathbf{M}\right\}$ such that in graph $\mathcal{G}_{m+1}^{\prime}$, if each matching $M \in \mathbf{M}$ is scheduled for a fraction $\beta^{\prime}(M)$ of the time, then edge $\left(a_{j-1}, a_{j}\right)$ is active for a total fraction $\alpha_{j}$ of the time for $j=1,2, \ldots, m$, where an edge is active whenever a matching containing it is scheduled. Note that the corollary to Lemma 1 in [11] applies to bipartite graphs, and hence to the graph $\mathcal{G}_{m+1}^{\prime}$, which is bipartite.

Now, for each independent set $I \in \mathbf{I}$ in graph $\mathcal{G}_{m}$, let $\beta(I)=\beta^{\prime}(M(I))$. From the above one-to-one correspondence between independent sets in graph $\mathcal{G}_{m}$ and matchings in graph $\mathcal{G}_{m+1}^{\prime}$, it follows that $\{\beta(I): I \in \mathbf{I}\}$ is a probability distribution, and under this distribution, each node $j$ in graph $\mathcal{G}_{m}$ is selected with a total probability $\alpha_{j}$.

The result follows.

Proof of Lemma 4. Consider a symmetric NE. Assume that $\beta(I)>0$ for some $I \neq I_{o}, I_{e}$. Now, WLOG, we can assume that $I$ is maximal. So there must exist nodes $j, j+3 \in I$ for some $j$ (this is because $I_{o}$ and $I_{e}$ are the only maximal independent sets in which there do not exist such $j, j+3$ ). Since $I$ is an independent set, $j+1, j+2 \notin I$. If $\alpha_{j+1}+\alpha_{j+2}+\beta(I)>1$, then each primary simultaneously offers bandwidth at neighboring nodes $\left(\alpha_{j}\right.$ and $\alpha_{j+1}$ or $\alpha_{j+2}$ and $\alpha_{j+3}$ or $\alpha_{j+1}$ and $\left.\alpha_{j+2}\right)$ with a positive probability. So:

$$
\alpha_{j+1}+\alpha_{j+2} \leq 1-\beta(I)<1
$$

Thus, either $\alpha_{j+1}<\frac{1}{2}$ or $\alpha_{j+2}<\frac{1}{2}$ (or both). WLOG, assume that $\alpha_{j+1}<\frac{1}{2}$.

Case (i): $\alpha_{j}+\alpha_{j+1}<1$

In this case, by (36), it follows that a primary $i$ can unilaterally increase the probability, $\alpha_{j+1}$, of offering bandwidth at node $j+1$, and continue to offer bandwidth with probability $\alpha_{l}$ for each node $l \neq j+1$ without conflicting with node $j$ or $j+2$. (Note that Lemma 9 guarantees that there exist $\beta(I)$ corresponding to the new set of node probabilities $\alpha_{l}$.) This will increase the primary's expected payoff at node $j+1$ and hence its total expected payoff, which contradicts the fact that primary $i$ 's original strategy was a best response.

Case (ii): $\alpha_{j}+\alpha_{j+1}=1$

Since $\alpha_{j+1}<\frac{1}{2}$, we have $\alpha_{j}>\frac{1}{2}$; thus, $\alpha_{j+1}<\alpha_{j}$. Now, a primary can unilaterally reduce $\alpha_{j}$ by a small amount and increase $\alpha_{j+1}$ by that amount, without conflicting with node $j+2$, by (36). This will increase the primary's total expected utility because:
1. by Lemma 3, the expected payoff of a primary at node $l$ is $(v-c)\left(1-w\left(q \alpha_{l}, n\right)\right)$ similar to the single location analysis in Section 3.3 (see (24)), and hence,

2. since $w(x, n)$ is an increasing function of $x$ [9] and $\alpha_{j+1}<\alpha_{j}$, the payoff at node $j+1((v-c)(1-$ $\left.\left.w\left(q \alpha_{j+1}, n\right)\right)\right)$ is higher than that at node $j((v-$ c) $\left.\left(1-w\left(q \alpha_{j}, n\right)\right)\right)$.

This is a contradiction.

ProOF OF ThEOREM 3. By Lemma 4, in any symmetric NE, each primary offers bandwidth at nodes in $I_{e}$ (respectively, $I_{o}$ ) w.p. $t$ (respectively, $1-t$ ) and at all other independent sets w.p. 0. First, we show that $t=0$ does not constitute a NE. Suppose primaries $\{1, \ldots, n\} \backslash i$ offer bandwidth only at the nodes in $I_{o}$, and at each node in $I_{o}$, play the single-location NE strategy $\psi($.$) in (6). If$ primary $i$ offers bandwidth at the nodes in $I_{o}$, then its payoff maximizing strategy would be to play any price in $[\tilde{p}, v]$ at each node in $I_{o}-$ this would give it a payoff of $\tilde{p}-c$ at each node in $I_{o}$. So primary $i$ 's total expected utility if it offers bandwidth at the nodes in $I_{o}$ is $\frac{m}{2}(\tilde{p}-c)=\frac{m}{2}(v-c)(1-w(q, n))$. Primary $i$ total payoff if it offers bandwidth at the nodes in $I_{e}$, and sets the price $v$ at each node in $I_{e}$ is $\frac{m}{2}(v-c)>\frac{m}{2}(v-c)(1-w(q, n))$. Thus, the strategy of offering bandwidth at the nodes in $I_{O}$ is not a best response. Hence, the symmetric strategy profile with $t=0$ is not a NE.

By symmetry, it follows that the symmetric strategy profile with $t=1$ is not a NE.

Now, consider a symmetric strategy profile with $0<$ $t<1$. Under this strategy profile, the payoff of each primary $i$ if it offers bandwidth at the nodes in $I_{o}$ (respectively, $\left.I_{e}\right)$ is $\frac{m}{2}(v-c)(1-w(q(1-t), n))$ (respectively, $\left.\frac{m}{2}(v-c)(1-w(q t, n))\right)$. These must be equal for $I_{e}$ and $I_{o}$ to both be best responses. That is:

$$
\frac{m}{2}(v-c)(1-w(q(1-t), n))=\frac{m}{2}(v-c)(1-w(q t, n))
$$

The above equality clearly holds for $t=\frac{1}{2}$, and it can be easily checked, using the fact that $w(x, n)$ is a strictly increasing function of $x$ [9], that $t=\frac{1}{2}$ is the unique solution.

Finally, we show that the symmetric strategy profile with $t=\frac{1}{2}$ is a NE. Suppose primaries $\{1, \ldots, n\} \backslash i$ offer bandwidth w.p. $t=\frac{1}{2}$ at $I_{o}$ and $I_{e}$, and at each node, play the single-location NE strategy in (6) with $\frac{q}{2}$ in place of $q$. Then the maximum expected utility of primary $i$ at a single node is equal for all nodes $1, \ldots, m$ and equals $(v-$ c) $\left(1-w\left(\frac{q}{2}, n\right)\right)$; also, it is attained when it sets any price in the range $\left[(v-c)\left(1-w\left(\frac{q}{2}, n\right)\right), v\right]$ at the node. Thus, the expected utility of primary $i$ is maximized if it offers bandwidth at any maximum independent set and sets a price in the above range at each node in that set. Note that $I_{o}$ and $I_{e}$ are the only two maximum independent sets. Hence, the strategy in which primary $i$ offers bandwidth w.p. $t=\frac{1}{2}$ at $I_{o}$ and $I_{e}$, and at each node plays the singlelocation NE strategy in (6) with $\frac{q}{2}$ in place of $q$ is a best response. 
Proof of Theorem 4. Similar to the proof of Theorem 3 , it can be shown that the symmetric strategy profile with $t=1$ is not a NE because if primaries other than $i$ offer bandwidth only at the nodes in $I_{e}$, then primary $i$ 's best response would be to offer bandwidth at only the nodes in $I_{o}$ and set a price of $v$ at each node in $I_{o}$. Now, consider the strategy profile with some $t$, $0<t<1$. The total expected payoff of each primary if it offers bandwidth only at the nodes in $I_{o}$ (respectively, $\left.I_{e}\right)$ is $\left(\frac{m+1}{2}\right)(v-c)(1-w(q(1-t), n))$ (respectively, $\left(\frac{m-1}{2}\right)(v-c)(1-w(q t, n))$. For $I_{o}$ and $I_{e}$ to be both best responses, these must be equal, that is:

$$
\begin{aligned}
& \left(\frac{m+1}{2}\right)(v-c)(1-w(q(1-t), n)) \\
= & \left(\frac{m-1}{2}\right)(v-c)(1-w(q t, n))
\end{aligned}
$$

Simplifying, the above condition is equivalent to $f_{4}(t)=$ 0, where:

$$
f_{4}(t)=2-(m+1) w(q(1-t), n)+(m-1) w(q t, n)
$$

Now, we have the following result, which we prove below.

LEMMA 10. $f_{4}(x)$ is a strictly increasing function of $x$ on $[0,1]$.

Also:

$$
f_{4}(0)=2-(m+1) w(q, n) \geq 0
$$

since $w(q, n) \leq \frac{2}{m+1}$. Thus, $f_{4}(t)>0$ for all $0<t<1$; so the condition $f_{4}(t)=0$ is not satisfied for any $0<t<1$. Thus, the symmetric strategy profile corresponding to $t$ is not a NE for any $t, 0<t<1$.

Finally, we show that the symmetric strategy profile with $t=0$ is a NE. Suppose primaries other than $i$ offer bandwidth only at $I_{o}$ and set the price at each node in $I_{O}$ according to the single-location NE strategy in (6). Then the maximum expected utility of primary $i$ at each node in $I_{o}$ equals $\tilde{p}-c$; also, it is attained when it sets any price in the range $[\tilde{p}, v]$ at the node. So the maximum total expected utility of primary $i$ if it offers bandwidth at the nodes in $I_{o}$ is $\left(\frac{m+1}{2}\right)(\tilde{p}-c)=\left(\frac{m+1}{2}\right)(v-c)(1-w(q, n))$. Also, the maximum total expected utility of primary $i$ if it offers bandwidth at the nodes in $I_{e}$ is attained when it sets a price of $v$ at each node in $I_{e}$ and equals $\left(\frac{m-1}{2}\right)(v-c)$ which is less than or equal to $\left(\frac{m+1}{2}\right)(v-c)(1-w(q, n))$ since $w(q, n) \leq \frac{2}{m+1}$. It follows that the strategy in which primary $i$ offers bandwidth only at $I_{o}$ and sets the price at each node in $I_{o}$ according to the single-location $\mathrm{NE}$ strategy in (6) is a best response. Hence, the symmetric strategy profile with $t=0$ is an NE.

PROOF OF LEMMA 10. It can be shown that [13] the derivative of $w(x, n)$ with respect to $x$ is given by:

$$
w^{\prime}(x, n)=(n-1)\left(\begin{array}{c}
n-2 \\
k-1
\end{array}\right) x^{k-1}(1-x)^{n-k-1} .
$$

Note that:

$$
w^{\prime}(x, n)>0, \forall 0<x<1
$$

Now,

$$
\begin{aligned}
f_{4}^{\prime}(x) & =(m-1) w^{\prime}(q x, n)(q)-(m+1) w^{\prime}(q(1-x), n)(-q) \\
& =q\left[(m-1) w^{\prime}(q x, n)+(m+1) w^{\prime}(q(1-x), n)\right] \\
& >0 \text { for } x \in(0,1),(\text { by }(38))
\end{aligned}
$$

So $f_{4}(x)$ is strictly increasing in $[0,1]$.

Proof of TheOrem 5. Similar to the proof of Theorem 3 , it can be shown that the symmetric strategy profiles with $t=0$ or $t=1$ are not NE.

Now, a necessary condition for the symmetric strategy profile with some $t, 0<t<1$, to be a NE is that the total expected payoffs that a primary gets at $I_{o}$ and $I_{e}$ are equal, which is equivalent to the condition $f_{4}(t)=0$, for the function $f_{4}(t)$ in (37). Now, we have the following result, which we prove below.

LEMMA 11. The function $f_{4}(x)$ in (37) has a unique root, say $t$, in $(0,1)$.

Similar to the proof of Theorem 3 , it can be shown that the symmetric strategy profile with $t$ equal to the root of $f_{4}(x)$ is a NE.

PROOF OF LEMMA 11. We have:

$$
\begin{aligned}
f_{4}(1) & =w(q, n)(m-1)-w(0, n)(m+1)+2 \\
& =w(q, n)(m-1)+2(\text { since } w(0, n)=0) \\
& >0 \\
f_{4}(0)= & w(0, n)(m-1)-w(q, n)(m+1)+2 \\
= & -w(q, n)(m+1)+2 \\
< & 0\left(\text { since } w(q, n)>\frac{2}{m+1} \text { by assumption }\right)
\end{aligned}
$$

Since $w(x, n)$ is continuous, so is $f_{4}(x)$ and hence by the intermediate value theorem [12], $f_{4}(x)$ has a root in $(0,1)$.

By Lemma $10, f_{4}(x)$ is strictly increasing in $[0,1]$ and hence can have at most one root in $(0,1)$.

The result follows.

\section{E Proofs of Results in Section 4.2}

Let $p_{i}=\left(p_{i,(j, l)}: j, l \in\{1, \ldots, m\}\right)$ denote the vector of prices that primary $i$ sets at the nodes in the grid, where $p_{i,(j, l)}$ is the price at node $V_{j l} \cdot p_{i,(j, l)}=\infty$ denotes that primary $i$ does not offer bandwidth at node $V_{j l}$. Let $u_{i,(j, l)}\left(p_{1}, \ldots, p_{n}\right)$ denote the revenue that primary $i$ gets at node $V_{j l}$ when the price vector chosen by primary $s$ is $p_{s}, s=1, \ldots, n$. Also, let $u_{i}\left(p_{1}, \ldots, p_{n}\right)$ be primary $i$ 's total revenue over all nodes.

\section{E.1 Even $m$}

Let $\Psi_{e}$ be the strategy in which a primary

- selects $I_{o}^{2}$ and $I_{e}^{2}$ w.p. $1 / 2$ each, whenever he has unused bandwidth, and

- decides the price at each node in the chosen independent set as per the distribution $\psi($.$) in Section 3.3,$ with $q$ replaced by $q / 2$ all through.

Let $\tilde{p}_{e}=v-w\left(\frac{q}{2}, n\right)(v-c)$.

THEOREM 9. The strategy profile in which each primary plays the strategy $\Psi_{e}$ is a Nash equilibrium. 
Proof. Suppose primaries $2, \ldots, n$ play the strategy $\Psi_{e}$. Similar to the single-location analysis, for every node $V_{j l}$ :

$$
E_{p_{2}, \ldots, p_{n}}\left\{u_{1,(j, l)}\left(p_{1}, \ldots, p_{n}\right)\right\} \begin{array}{ll}
=\tilde{p}_{e}-c & \text { if } p_{1,(j, l)} \in\left[\tilde{p}_{e}, v\right] \\
<\tilde{p}_{e}-c & \text { if } p_{1,(j, l)}<\tilde{p}_{e}
\end{array}
$$

Note that $I_{o}^{2}$ and $I_{e}^{2}$ are maximum independent sets, and $\left|I_{o}^{2}\right|=\left|I_{e}^{2}\right|=\frac{m^{2}}{2}$. So if primary 1 plays $\Psi_{e}$, it gets a total expected payoff of $\frac{m^{2}}{2}\left(\tilde{p}_{e}-c\right)$. Also, if it offers bandwidth at any independent set $I$, it gets a payoff of at most $|I|\left(\tilde{p}_{e}-c\right) \leq \frac{m^{2}}{2}\left(\tilde{p}_{e}-c\right)$, since the maximum expected payoff at any node is $\tilde{p}_{e}-c$. Hence, $\Psi_{e}$ is a best response.

\section{E.2 Odd $m$}

\section{E.2.1 The Case $w(q, n) \leq \frac{2}{m^{2}+1}$}

Let $\Psi_{o, 1}$ be the strategy in which a primary

- offers unused bandwidth at only the nodes in $I_{o}^{2}$, and

- selects the price at each node in $I_{o}^{2}$ according to the d.f. $\psi($.$) in Section 3.3.$

THEOREM 10. The strategy profile in which each primary plays $\Psi_{o, 1}$ is a Nash equilibrium.

PROOF. Let $p_{2}, \ldots, p_{n}$ be distributed according to $\Psi_{o, 1}$. Let $\tilde{p}$ be as in (3). From the single-location Nash equilibrium analysis, if $p_{1}$ is distributed according to $\Psi_{o, 1}$ :

$$
E_{p_{2}, \ldots, p_{n}}\left\{u_{1}\left(p_{1}, \ldots, p_{n}\right)\right\}=\left(\frac{m^{2}+1}{2}\right)(\tilde{p}-c)
$$

Since at any given node $V_{j l}$ in $I_{o}^{2}, p_{1,(j, l)} \sim \psi($.$) is a$ best response to $p_{i,(j, l)} \sim \psi(),. i=2, \ldots, n$, the above is the maximum utility obtainable if primary 1 offers bandwidth at the nodes in $I_{o}^{2}$. Now, suppose primary 1 offers bandwidth at nodes in some other independent set $I \neq I_{o}^{2}$. Since $I_{o}^{2}$ is the unique maximum independent set of size $\frac{m^{2}+1}{2}$ :

$$
|I| \leq \frac{m^{2}+1}{2}-1=\frac{m^{2}-1}{2}
$$

So:

$$
\begin{aligned}
u_{1}\left(p_{1}, \ldots, p_{n}\right) & \leq|I|(v-c) \\
& \leq\left(\frac{m^{2}-1}{2}\right)(v-c) \\
& \leq\left(\frac{m^{2}+1}{2}\right)(\tilde{p}-c)
\end{aligned}
$$

where the last inequality holds if and only if $w(q, n) \leq$ $\frac{2}{m^{2}+1}$ (this follows by algebraic simplification).

Since $w(q, n) \leq \frac{2}{m^{2}+1}$ by assumption, it follows that $p_{1} \sim \Psi_{o, 1}$ is a best response to $p_{2}, \ldots, p_{n} \sim \Psi_{o, 1}$.

\section{E.2.2 The Case $w(q, n)>\frac{2}{m^{2}+1}$}

We first prove a simple property of independent sets in $\mathcal{G}_{m, m}$. To that end, we first consider a linear graph. Recall that $\mathcal{G}_{m}$ denotes a graph with $m$ nodes $1, \ldots, m$, with edges $(j, j+1), j=1, \ldots, m-1$. Let $I_{l}^{m}=\{1,3, \ldots, m\}$ and $I_{s}^{m}=\{2,4, \ldots, m-1\}$.
LEMMA 12. Let $m \geq 3$ be odd. In graph $\mathcal{G}_{m}$, let I be an independent set with $m_{l}$ nodes from $I_{l}^{m}$ and $m_{s}$ nodes from $I_{s}^{m}$. Then:

$$
m_{l}+m_{s}\left(\frac{m+1}{m-1}\right) \leq \frac{m+1}{2}
$$

ProOF. We prove the result by induction. For $m=3$, the only possibilities are $I=\{1\},\{2\},\{3\},\{1,3\}$. In each case, (39) clearly holds.

Suppose (39) is true for $m$, where $m$ is odd. We will now prove the result for $m+2$. Consider an independent set $I$ in graph $\mathcal{G}_{m+2}$.

Case 1: $m+1 \notin I$. Assume, WLOG, that $m+2 \in I$. In addition, suppose there are $m_{l}$ and $m_{s}$ nodes from $I_{l}^{m}$ and $I_{s}^{m}$ respectively in $I$. Hence, there are $\left(m_{l}+1\right)$ and $m_{s}$ nodes from $I_{l}^{m+2}$ and $I_{s}^{m+2}$ respectively in $I$. We have:

$$
\begin{aligned}
& m_{l}+1+m_{s}\left(\frac{m+3}{m+1}\right) \\
= & \frac{m\left(m_{l}+m_{s}\right)+\left(m_{l}+3 m_{s}\right)+(m+1)}{m+1} \\
= & \frac{\left[m\left(m_{l}+m_{s}\right)+\left(m_{s}-m_{l}\right)\right]+2\left(m_{l}+m_{s}\right)+(m+1)}{m+1} \\
\leq & \frac{\frac{m^{2}-1}{2}+2\left(m_{l}+m_{s}\right)+(m+1)}{m+1}
\end{aligned}
$$

(by (39), which is true by induction hypothesis)

$$
\begin{aligned}
& \leq \frac{\frac{m^{2}-1}{2}+2\left(\frac{m+1}{2}\right)+(m+1)}{m+1} \\
& =\frac{m+3}{2}
\end{aligned}
$$

which proves the result. The last inequality follows from the fact that $m_{l}+m_{s}$ is the size of an independent set in the subgraph $\{1, \ldots, m\}$ of $\mathcal{G}_{m+2}$ and hence $m_{l}+m_{s} \leq \frac{m+1}{2}$.

Case 2: $m+1 \in I$. Again, suppose there are $m_{l}$ and $m_{s}$ nodes from $I_{l}^{m}$ and $I_{s}^{m}$ respectively in $I$. Since $m+1 \in I$, $m \notin I$. So, since $\{1,3, \ldots, m\}$ is the only independent set of size $\frac{m+1}{2}$ in $\{1, \ldots, m\}, I \backslash\{m+1\}$ is an independent set of size at most $\frac{m-1}{2}$, i.e.:

$$
m_{l}+m_{s} \leq \frac{m-1}{2}
$$

Now:

$$
\begin{aligned}
& m_{l}+\left(m_{s}+1\right)\left(\frac{m+3}{m+1}\right) \\
= & \frac{m\left(m_{l}+m_{s}\right)+\left(m_{l}+3 m_{s}\right)+(m+3)}{m+1} \\
= & \frac{\left[m\left(m_{l}+m_{s}\right)+\left(m_{s}-m_{l}\right)\right]+2\left(m_{l}+m_{s}\right)+(m+3)}{m+1} \\
\leq & \frac{\frac{m^{2}-1}{2}+2\left(\frac{m-1}{2}\right)+(m+3)}{m+1} \\
= & \frac{(\text { by induction hypothesis and }(40))}{2}
\end{aligned}
$$


This proves the result for $m+2$ and hence the proof is complete by induction.

Now, using the above lemma, we prove a property of $\mathcal{G}_{m, m}$.

LEMMA 13. Let $m \geq 3$ be odd. In graph $\mathcal{G}_{m, m}$, let I be an independent set with $m_{l}$ nodes from $I_{o}^{2}$ and $m_{s}$ nodes from $I_{e}^{2}$. Then:

$$
m_{l}+m_{s}\left(\frac{m^{2}+1}{m^{2}-1}\right) \leq \frac{m^{2}+1}{2}
$$

Proof. We construct the linear graph $\mathcal{G}_{m^{2}}$ from $\mathcal{G}_{m, m}$ by deleting some edges, as follows. Retain all horizontal edges (see part (b) of Fig. 3). Retain only the following vertical edges: $\left(V_{1, m}, V_{2, m}\right),\left(V_{2,1}, V_{3,1}\right),\left(V_{3, m}, V_{4, m}\right)$, $\left(V_{4,1}, V_{5,1}\right), \ldots,\left(V_{m-2, m}, V_{m-1, m}\right),\left(V_{m-1,1}, V_{m, 1}\right)$. Delete the rest of the vertical edges. Note that the resulting graph is $\mathcal{G}_{m^{2}}$, the linear graph with $m^{2}$ nodes, and $m^{2}$ is odd. Also, the nodes in $I_{o}^{2}$ and $I_{e}^{2}$ in $\mathcal{G}_{m, m}$ constitute the independent sets $I_{l}^{m^{2}}$ and $I_{s}^{m^{2}}$ in $\mathcal{G}_{m^{2}}$.

Now, since $\mathcal{G}_{m^{2}}$ is obtained from $\mathcal{G}_{m, m}$ by deleting some edges, $I$ is an independent set in $\mathcal{G}_{m^{2}}$ as well. So by (39) in Lemma 12, we get (41).

Now, similar to Lemma 11, we get the following result using the fact that $w(q, n)>\frac{2}{m^{2}+1}$.

LEMMA 14.

$$
f_{5}(x)=w(q x, n)\left(m^{2}-1\right)-w(q(1-x), n)\left(m^{2}+1\right)+2
$$

has a unique root, say $t_{2}$, in $(0,1)$.

Let $\Psi_{o, 2}$ be the strategy in which, whenever a primary has unused bandwidth,

- it selects $I_{e}^{2}$ and $I_{o}^{2}$ w.p. $t_{2}$ and $1-t_{2}$ respectively, where $t_{2}$ is the unique root in $(0,1)$ of $(42)$, and

- selects the price at each node as per the distribution $\psi($.$) in Section 3.3, with q$ replaced by $q t_{2}\left(q\left(1-t_{2}\right)\right.$ resp.) whenever he selects $I_{e}^{2}\left(I_{o}^{2}\right.$ resp.).

Let $\quad \tilde{p}_{l}=v-w\left(q\left(1-t_{2}\right), n\right)(v-c)$ and $\tilde{p}_{s}=v-$ $w\left(q t_{2}, n\right)(v-c)$. From the fact that $f_{5}\left(t_{2}\right)=0$ (see Lemma 14), it follows that:

$$
\left(\frac{m^{2}+1}{2}\right)\left(\tilde{p}_{l}-c\right)=\left(\frac{m^{2}-1}{2}\right)\left(\tilde{p}_{s}-c\right)
$$

THEOREM 11. The strategy profile in which each primary plays the strategy $\Psi_{o, 2}$ is a Nash equilibrium.

PROOF. Suppose primaries $2, \ldots, n$ play $\Psi_{o, 2}$. Similar to the single-location Nash equilibrium analysis, if at a node $V_{j l} \in I_{o}^{2}$, primary 1 offers a price $p_{1,(j, l)} \in\left[\tilde{p}_{l}, v\right]$, then:

$$
E_{p_{2}, \ldots, p_{n}}\left\{u_{1,(j, l)}\left(p_{1}, \ldots, p_{n}\right)\right\}=\tilde{p}_{l}-c
$$

If primary 1 offers a price $p_{1,(j, l)}<\tilde{p}_{l}$, then:

$$
u_{1,(j, l)}\left(p_{1}, \ldots, p_{n}\right)<\tilde{p}_{l}-c
$$

Similar results hold for nodes $V_{j l} \in I_{e}^{2}$. Now, if primary 1 offers bandwidth at the nodes in $I_{o}^{2}$ (respectively, $I_{e}^{2}$ ) and offers a price $p_{1,(j, l)} \in\left[\tilde{p}_{l}, v\right]$ (respectively, $p_{1,(j, l)} \in$ $\left[\tilde{p}_{s}, v\right]$ ) at each node $V_{j l} \in I_{o}^{2}$ (respectively, $V_{j l} \in I_{e}^{2}$ ), then its expected payoff is $\left(\frac{m^{2}+1}{2}\right)\left(\tilde{p}_{l}-c\right)$ (respectively, $\left(\frac{m^{2}-1}{2}\right)\left(\tilde{p}_{s}-c\right)=\left(\frac{m^{2}+1}{2}\right)\left(\tilde{p}_{l}-c\right)$ by (43)). Since $\Psi_{o, 2}$ randomizes among these strategies, its expected payoff is $\left(\frac{m^{2}+1}{2}\right)\left(\tilde{p}_{l}-c\right)$.

Now, we will show that the expected total revenue under any strategy is at most $\left(\frac{m^{2}+1}{2}\right)\left(\tilde{p}_{l}-c\right)$, which will show that $\Psi_{o, 2}$ is a best response.

Consider a strategy which offers bandwidth at an independent set $I$ with $m_{l}$ nodes in $I_{o}^{2}$ and $m_{s}$ nodes in $I_{e}^{2}$. As shown above, the maximum expected revenue at a node in $I_{o}^{2}$ (respectively, $\left.I_{e}^{2}\right)$ is $\left(\tilde{p}_{l}-c\right)$ (respectively, $\left(\tilde{p}_{s}-c\right)$ ). So the revenue of this strategy is at most:

$$
\begin{aligned}
& m_{l}\left(\tilde{p}_{l}-c\right)+m_{s}\left(\tilde{p}_{s}-c\right) \\
= & m_{l}\left(\tilde{p}_{l}-c\right)+m_{s}\left(\frac{m^{2}+1}{m^{2}-1}\right)\left(\tilde{p}_{l}-c\right)(\text { by }(43)) \\
= & \left(\tilde{p}_{l}-c\right)\left[m_{l}+m_{s}\left(\frac{m^{2}+1}{m^{2}-1}\right)\right] \\
\leq & \left(\tilde{p}_{l}-c\right)\left(\frac{m^{2}+1}{2}\right) \text { (by Lemma 13) }
\end{aligned}
$$

Thus, $\Psi_{o, 2}$ is a best response and the strategy profile in which each primary plays $\Psi_{o, 2}$ is a Nash equilibrium.

\section{F Proofs of Results in Section 5}

ProOf OF THEOREM 6. Let $u_{i}^{O S}$ be the expected payoff that primary $i$ receives in the one-shot game Nash equilibrium, in which each primary plays the strategy $\psi($.$) in (6).$ Let $u_{i}^{P D}$ be his expected payoff in each stage game of the repeated game when all primaries play the pre-deviation strategy in the above Nash reversion strategy. Also, let $u_{i}^{\text {sup }}$ be the supremum over the possible expected payoffs that primary $i$ can get in a single stage game by using any strategy, when all primaries played the pre-deviation strategy in all slots until the previous stage game, and primaries other than $i$ play the pre-deviation strategy in the current stage game.

It can be shown that a sufficient condition for the above Nash reversion strategy to be a SPNE is (the proof is similar to that of (12.AA.1) in [1]):

$$
u_{i}^{\text {sup }}+\frac{q \delta}{1-\delta} u_{i}^{O S} \leq u_{i}^{P D}+\frac{q \delta}{1-\delta} u_{i}^{P D}
$$

Note that the left-hand side is primary $i$ 's maximum (discounted) payoff starting from a given slot if he deviates from the pre-deviation strategy, and the right-hand side is the payoff if he does not deviate. The factor $q$ appears in the second term on either side to account for the fact that primary $i$ would have free bandwidth in each future slot with probability $q$. So if condition (44) is met, primary $i$ would not deviate from its pre-deviation strategy.

Next, we compute $u_{i}^{P D}, u_{i}^{O S}$ and $u_{i}^{\text {sup }}$. Let $E(n, q)$ be the event that when primary 1 has bandwidth available, and sets $p_{1}=v$, and each primary $i=2, \ldots, n$ sets $p_{i}=$ $v$ (provided he has bandwidth available), then primary 1 is among the $k$ randomly selected primary from whom 
bandwidth is bought by the $k$ secondaries. Let the random variable $A$ be the number of primaries from $2, \ldots, n$ who have bandwidth available. $A$ has a binomial distribution with parameters $n-1$ and $q$. Conditioning on $A$ :

$$
P(E(n, q))=\sum_{i=0}^{n-1} P(E(n, q) \mid A=i) P(A=i)
$$

Now,

$$
P(E(n, q) \mid A=i)= \begin{cases}1, & i<k \\ \left(\frac{k}{i+1}\right), & i \geq k\end{cases}
$$

Putting these along with $P(A=i)=\left(\begin{array}{c}n-1 \\ i\end{array}\right) q^{i}(1-$ $q)^{n-1-i}$ into the expression for $P(E(n, q))$, we get:

$$
\begin{aligned}
P(E(n, q))= & \sum_{i=0}^{k-1}\left(\begin{array}{c}
n-1 \\
i
\end{array}\right) q^{i}(1-q)^{n-1-i} \\
& +\sum_{i=k}^{n-1}\left(\frac{k}{i+1}\right)\left(\begin{array}{c}
n-1 \\
i
\end{array}\right) q^{i}(1-q)^{n-1-i} \\
= & 1-w(q, n)+\beta(q, n)
\end{aligned}
$$

where $w(q, n)$ is as in (2) and $\beta(q, n)$ is as in (12).

Now, $u_{1}^{O S}=(v-c)(1-w(q, n))$ as derived in Section 3. Also, by definition of the event $E(n, q), u_{1}^{P D}=$ $(v-c) P(E(n, q))$. Finally, since the strategy $p_{1}=v-\varepsilon$ yields the payoff $v-\varepsilon-c$ for every $\varepsilon>0$, we have $u_{1}^{\text {sup }}=(v-c)$. Substituting these expressions into (44), putting the value of $P(E(n, q))$ from (45), and some algebraic simplification, yields that the above Nash reversion strategy is an SPNE if $\delta \geq \delta_{t}$.

\section{G Proofs of Results in Section 6}

Proof of Theorem 7. Let $p_{1}, \tilde{p}_{2} \leq p_{1} \leq v$, be fixed. If $p_{1}<p_{2}$, then primary 1's bandwidth is sold, and if $p_{1}>p_{2}$, then primary 1 's bandwidth is sold iff primary 2 has no unused bandwidth. So primary 1's utility is given by:

$$
u_{1}\left(p_{1}, p_{2}\right)= \begin{cases}p_{1}-c & \text { if } p_{1}<p_{2} \\ \left(p_{1}-c\right)\left(1-q_{2}\right) & \text { if } p_{1}>p_{2}\end{cases}
$$

Suppose primary 2 uses the mixed strategy $\psi_{2}($.$) . Note$ that for fixed $p_{1}$, since $\psi_{2}($.$) is continuous, the event p_{2}=$ $p_{1}$ has zero probability. Hence,

$$
\begin{aligned}
E_{p_{2}\left[u_{1}\left(p_{1}, p_{2}\right)\right]=} & \left(p_{1}-c\right) P\left(p_{2}>p_{1}\right) \\
& +\left(p_{1}-c\right)\left(1-q_{2}\right) P\left(p_{2}<p_{1}\right) \\
= & \left(p_{1}-c\right)\left[1-\psi_{2}\left(p_{1}\right)\right] \\
& +\left(p_{1}-c\right)\left(1-q_{2}\right) \psi_{2}\left(p_{1}\right) \\
= & \left(p_{1}-c\right)\left(1-q_{2} \psi_{2}\left(p_{1}\right)\right) \\
= & \left(p_{1}-c\right)\left(1-q_{2} \frac{1}{q_{2}}\left(\frac{p_{1}-\tilde{p}_{2}}{p_{1}-c}\right)\right) \\
= & \left(p_{1}-c\right)\left(\frac{\tilde{p}_{2}-c}{p_{1}-c}\right) \\
= & \tilde{p}_{2}-c
\end{aligned}
$$

Thus, primary 1's expected payoff is constant, equal to $\tilde{p}_{2}-c$, for $\tilde{p}_{2} \leq p_{1} \leq v$. For $p_{1}<\tilde{p}_{2}$, primary 1's payoff is at most $p_{1}-c<\tilde{p}_{2}-c$. So $p_{1}$ is a best response to $\psi_{2}($.$) for each p_{1} \in\left[\tilde{p}_{2}, v\right]$. Since $\psi_{1}($.$) randomizes over$ $p_{1} \in\left[\tilde{p}_{2}, v\right], \psi_{1}($.$) is a best response to \psi_{2}($.$) .$

Now, let $p_{2}, \tilde{p}_{2} \leq p_{2}<v$, be fixed. Similar to (46):

$$
u_{2}\left(p_{1}, p_{2}\right)= \begin{cases}p_{2}-c & \text { if } p_{2}<p_{1} \\ \left(p_{2}-c\right)\left(1-q_{1}\right) & \text { if } p_{2}>p_{1}\end{cases}
$$

Suppose primary 1 uses the mixed strategy $\psi_{1}($.$) . Since$ $\psi_{1}\left(p_{1}\right)$ is continuous on $\tilde{p}_{2} \leq p_{1}<v, P\left(p_{1}=p_{2}\right)=0$ for $\tilde{p}_{2} \leq p_{2}<v$. So for $\tilde{p}_{2} \leq p_{2}<v$ :

$$
\begin{aligned}
E_{p_{1}}\left(u_{2}\left(p_{1}, p_{2}\right)\right)= & \left(p_{2}-c\right) P\left(p_{1}>p_{2}\right) \\
& +\left(p_{2}-c\right)\left(1-q_{1}\right) P\left(p_{1}<p_{2}\right) \\
= & \left(p_{2}-c\right)\left(1-\psi_{1}\left(p_{2}\right)\right) \\
& +\left(p_{2}-c\right)\left(1-q_{1}\right) \psi_{1}\left(p_{2}\right) \\
= & \left(p_{2}-c\right)\left(1-q_{1} \psi_{1}\left(p_{2}\right)\right) \\
= & \left(p_{2}-c\right)\left(1-q_{1} \frac{1}{q_{1}}\left(\frac{p_{2}-\tilde{p}_{2}}{p_{2}-c}\right)\right) \\
= & \left(p_{2}-c\right)\left(\frac{\tilde{p}_{2}-c}{p_{2}-c}\right) \\
= & \tilde{p}_{2}-c
\end{aligned}
$$

For $p_{2}<\tilde{p}_{2}$ :

$$
u_{2}\left(p_{1}, p_{2}\right) \leq p_{2}-c<\tilde{p}_{2}-c
$$

Now, let $p_{2}=v$. Note that $P\left(p_{1}=v\right)=1-\frac{q_{2}}{q_{1}}>0$. If $p_{1}=v$, then primary 2's payoff is $(v-c)$ if primary 1 has no unused bandwidth or if primary 1 has unused bandwidth and the secondary (randomly) selects primary 2 to buy bandwidth from, and 0 otherwise. Thus, if $p_{1}=v$, then the probability that primary 2's payoff is $(v-c)$ is $1-q_{1}+\frac{q_{1}}{2}=1-\frac{q_{1}}{2}$. Thus,

$$
u_{2}\left(p_{1}, v\right)= \begin{cases}(v-c)\left(1-q_{1}\right) & \text { if } p_{1}<v \\ (v-c)\left(1-\frac{q_{1}}{2}\right) & \text { if } p_{1}=v\end{cases}
$$


Hence:

$$
\begin{aligned}
E_{p_{1}}\left(u_{2}\left(p_{1}, v\right)\right) \\
=\quad(v-c)\left(1-q_{1}\right) P\left(p_{1}<v\right) \\
\quad+(v-c)\left(1-\frac{q_{1}}{2}\right) P\left(p_{1}=v\right) \\
=\quad(v-c)\left(1-q_{1}\right) \psi_{1}(v-) \\
\quad+(v-c)\left(1-\frac{q_{1}}{2}\right)\left(1-\frac{q_{2}}{q_{1}}\right) \\
=\quad(v-c)\left(1-q_{1}\right) \frac{1}{q_{1}}\left(\frac{v-\tilde{p}_{2}}{v-c}\right) \\
\quad+(v-c)\left(1-\frac{q_{1}}{2}\right)\left(1-\frac{q_{2}}{q_{1}}\right) \\
\leq \quad \frac{1-q_{1}}{q_{1}}\left(v-\tilde{p}_{2}\right)+(v-c)\left(1-\frac{q_{2}}{q_{1}}\right) \\
=\frac{1}{q_{1}}\left(v-q_{2}(v-c)-\tilde{p}_{2}+q_{1} \tilde{p}_{2}-q_{1} c\right) \\
=\frac{1}{q_{1}}\left(q_{1}\left(\tilde{p}_{2}-c\right)\right) \\
=\frac{\tilde{p}_{2}-c}{}
\end{aligned}
$$

By (47), (48) and (49), each $p_{2}, \tilde{p}_{2} \leq p_{2}<v$, is a best response.

Since $\psi_{2}($.$) randomizes over p_{2} \in\left[\tilde{p}_{2}, v\right), \psi_{2}($.$) is a$ best response to $\psi_{1}($.$) .$

Thus, $\left(\psi_{1}(),. \psi_{2}().\right)$ is a Nash equilibrium.

ProOF OF LEMMA 5. Let

$$
f_{2}(x)=(x-c)(\bar{v}-x)-\frac{(1-q)^{n-1}(\bar{v}-c)^{2}}{4}
$$

It is sufficient to show that the quadratic $f_{2}(x)$ has exactly one root in $\left(c, v_{T}\right)$, the other root being in $\left(v_{T}, \bar{v}\right)$. Now,

$$
\begin{gathered}
f_{2}(c)=-\frac{(1-q)^{n-1}(\bar{v}-c)^{2}}{4}<0 \\
f_{2}\left(v_{T}\right)=\left(v_{T}-c\right)\left(\bar{v}-v_{T}\right)-\frac{(1-q)^{n-1}(\bar{v}-c)^{2}}{4} \\
=\frac{(\bar{v}-c)^{2}}{4}\left\{1-(1-q)^{n-1}\right\}>0
\end{gathered}
$$

So, since $f_{2}(x)$ is continuous, by the intermediate value theorem [12], it has a root in $\left(c, v_{T}\right)$. Also,

$$
f_{2}(\bar{v})=-\frac{(1-q)^{n-1}(\bar{v}-c)^{2}}{4}<0
$$

So again, by the intermediate value theorem, $f_{2}(x)$ has a root in $\left(v_{T}, \bar{v}\right)$, and the result follows.

ProOF OF LEMMA 6. Let

$$
f_{3}(x)=\frac{4(x-c)(\bar{v}-\underline{v})}{(1-q)^{n-1}(\bar{v}-c)^{2}}
$$

Then:

$$
\begin{aligned}
f_{3}\left(\tilde{p}_{1}\right) & =\frac{4\left(\tilde{p}_{1}-c\right)(\bar{v}-\underline{v})}{(1-q)^{n-1}(\bar{v}-c)^{2}} \\
& =\frac{\bar{v}-\underline{v}}{\bar{v}-\tilde{p}_{1}}(\text { by }(20)) \\
& <1\left(\text { since } \tilde{p}_{1}<\underline{v}<\bar{v}\right) \\
f_{3}(\underline{v})= & \frac{4(\underline{v}-c)(\bar{v}-\underline{v})}{(1-q)^{n-1}(\bar{v}-c)^{2}} \\
= & \frac{(\underline{v}-c)(\bar{v}-\underline{v})}{\left(\tilde{p}_{1}-c\right)\left(\bar{v}-\tilde{p}_{1}\right)}(\operatorname{by}(20))
\end{aligned}
$$

So $f_{3}(\underline{v})>1$ iff:

$$
\begin{array}{r}
(\underline{v}-c)(\bar{v}-\underline{v})>\left(\tilde{p}_{1}-c\right)\left(\bar{v}-\tilde{p}_{1}\right) \\
\Leftrightarrow\left(\underline{v}-\tilde{p}_{1}\right)\left(\bar{v}+c-\underline{v}-\tilde{p}_{1}\right)>0
\end{array}
$$

which is true because $\underline{v}-\tilde{p}_{1}>0$ and $\bar{v}+c>2 \underline{v}>\underline{v}+\tilde{p}_{1}$. Thus, $f_{3}\left(\tilde{p}_{1}\right)<1$ and $f_{3}(\underline{v})>1$. Since $f_{3}(x)$ is continuous, by the intermediate value theorem [12], there exists $\tilde{p}_{2}$, where $\tilde{p}_{1}<\tilde{p}_{2}<\underline{v}$, such that $f_{3}\left(\tilde{p}_{2}\right)=1$.

Also, since $f_{3}($.$) is of the form:$

$$
f_{3}(x)=a(x-c)
$$

where $a>0, f_{3}$ is one-to-one and hence $\tilde{p}_{2}$ is unique. 